\title{
GLAD!
}

Revue sur le langage, le genre, les sexualités

$07 \mid 2019$

Varia

\section{Le langage de la diversité}

The Language of Diversity

\section{Sara Ahmed}

Traducteur : Noémie Grunenwald

\section{CpenEdition}

\section{Journals}

Édition électronique

URL : http://journals.openedition.org/glad/1647

DOI : 10.4000/glad.1647

ISSN : 2551-0819

Éditeur

Association GSL

\section{Référence électronique}

Sara Ahmed, "Le langage de la diversité », GLAD! [En ligne], 07 | 2019, mis en ligne le 05 décembre 2019, consulté le 17 décembre 2020. URL : http://journals.openedition.org/glad/1647 ; DOI : https:// doi.org/10.4000/glad. 1647

Ce document a été généré automatiquement le 17 décembre 2020.

\section{c) ()요}

La revue GLAD! est mise à disposition selon les termes de la Licence Creative Commons Attribution Pas d'Utilisation Commerciale - Pas de Modification 4.0 International. 


\title{
Le langage de la diversité
}

\author{
The Language of Diversity
}

\section{Sara Ahmed}

Traduction : Noémie Grunenwald

\section{RÉFÉRENCE}

"The Language of Diversity", in On Being Included, Sara Ahmed, pp. 51-82. Copyright, 2012, Duke University Press. All rights reserved.

"The Language of Diversity", in On Being Included, Sara Ahmed, pp. 51-82. Copyright, 2012, Duke University Press. All rights reserved. Republished by permission of the copyright holder. www.dukeupress.edu. The French translation is by permission - and is not subject to the journal's cc license. All requests for reuse must be through the originating publisher.

Ce travail de traduction a en partie été réalisé grâce au soutien financier du Laboratoire Triangle apporté à FELiCiTE.

A titre exceptionnel, la revue GLAD! a accepté de publier ce texte selon une licence fermée et propriétaire qui s'écarte des principes de libre circulation du savoir, que revendique et défend la revue. Ce texte sur les dynamiques de la « diversité » dans les universités étasuniennes nous semble important de par sa résonance critique avec le contexte français. C'est pourquoi nous avons choisi, après de nombreuses discussions, de le publier malgré sa licence propriétaire.

1 Les professionnel•les de la diversité sont confronté•es à des institutions réfractaires à leur travail, bien que ce soient ces mêmes institutions qui les emploient pour « créer de la diversité ». Cette réticence est souvent décrite à travers la métaphore du mur de briques. Le travail de la diversité peut être d'autant plus frustrant que les insatisfactions qu'il engendre sont difficiles à expliquer: les travailleur•euses de la 
diversité rencontrent des obstacles qui sont souvent invisibles aux autres personnes avec qui els travaillent. La frustration n'est en aucun cas la seule façon dont les professionnel•les de la diversité subissent l'institutionnalité, mais la répétition des situations de frustration permet de révéler comment le travail de la diversité peut prendre la forme de confrontations répétées avec ce qui n'avance pas, et qui de toute évidence n'avancera pas.

2 Mais en même temps, la diversité en tant que terme semble omniprésente. Comme le note Nirmal Puwar: "Le langage de la diversité est aujourd'hui adopté en différents endroits comme un mantra sacré. On nous dit que la diversité est une bonne chose pour nous. Qu'elle construit une société multiculturelle plus riche.» (Puwar 2004: 1) Le langage de la diversité apparait bien entendu dans les déclarations officielles (dans des déclarations de mission, des proclamations de règlements sur l'égalité, des brochures, des slogans) et en tant que répertoire d'images (dans les collages de visages souriants de différentes couleurs) qui sont facilement reconnaissables en tant qu'images de la diversité. Diversité peut être employé comme adjectif, comme un moyen de décrire une organisation, une qualité ou un attribut d'une organisation. Le langage de la diversité peut également être utilisé de façon normative, comme une expression des priorités, des valeurs ou des engagements d'une organisation. Les usages normatifs et descriptifs de la diversité ne sont pas sans lien. Gavan Titley et Alana Lentin suggèrent que «l'apparente descriptivité de la diversité est un aspect central de son caractère normatif: la diversité implique de se baser sur des valeurs pour créer un projet de transformation vers l'irréductible et l'irrévocable.» (Titley \& Lentin 2008: 11) Après tout, les descriptions ne sont pas neutres: le fait de décrire une organisation comme étant diverse indique également les valeurs de cette organisation (même s'il n'est pas évident en soi de savoir à quoi se réfère la valeur de la diversité, comme je le montrerai en temps utile). Mon but dans ce [texte] est d'explorer ce qui pourrait de prime abord apparaitre comme un paradoxe entre, d'une part, les usages routiniers du langage de la diversité par les institutions et, d'autre part, l'expérience vécue par de nombreux•ses professionnel•les d'une résistance institutionnelle à la diversité qui se transforme en routine. Nous pouvons transformer ce paradoxe en question: de quelle manière la volonté institutionnelle à l'égard de la diversité ${ }^{1}$ est-elle liée au mur de l'institution? Ou plus simplement : comment la volonté se transforme-t-elle en mur?

3 Pour répondre à ces questions, nous devons identifier comment s'implante le mot diversité, comment la diversité s'intègre à une description routinière. Le terme diversité a été entendu comme un terme de substitution de mots plus anciens tels que égalité des chances ou antiracisme $e^{2}$. Nous ne pouvons pas expliquer ces changements de vocabulaire simplement du point de vue d'un développement interne du secteur de l'égalité, dans lequel le terme diversité est d'ailleurs largement soupçonné d'être une importation. On en parle même comme d'un terme étranger. Les critiques suggèrent en particulier que l'usage en hausse du langage de la diversité reflète la propagation d'un discours managérial étasunien (Kandola \& Fullerton 1984 ; Deem \& Ozga 1997 ; Kirton \& Greene 2000 ; Benschop 2001 ; Lorbiecki 2001 ; Blackmore \& Sachs 2003). L'usage de diversité au sein des universités a été traité comme un symptôme de ce que les universitaires ont appelé la " privatisation de l'université », comme l'illustre le titre d'un chapitre du livre de Mike Hill (2004) sur la blanchité : « la diversité de la multiversité ».

4 Le passage au langage de la diversité pourrait ainsi être interprété en termes de marché : la diversité possède une valeur commerciale et peut être utilisée non 
seulement comme un moyen de vendre l'université, mais aussi de la transformer en marché. D'autres ont qualifié ceci de "modèle Benetton » de la diversité, par lequel la diversité devient un style esthétique ou un moyen de "relooker " une organisation (Lury 2000: 147; Titley \& Lentin 200833). Peut-être en raison de la généralisation d'arguments dignes d'analyses de rentabilité pour justifier la diversité au sein d'organisation des secteurs publics ou privés, la diversité a été plus précisément identifiée comme un terme de management. La diversité devient quelque chose qui doit être géré et valorisé en tant que ressource humaine. Des chercheur•euses ont indiqué que l'accent managérial donné à la diversité a pour effet d'individualiser les différences et de dissimuler la perpétuation des inégalités systémiques au sein de l'université. Pour Deem et Ozga, le mot diversité invoque la différence mais ne suscite pas forcément l'engagement dans l'action ou dans la justice redistributive (Deem \& Ozga 1997 : 33). Par extension, ce qui est problématique avec la diversité, c'est le fait qu'elle puisse être " coupée » des programmes qui cherchent à s'attaquer aux inégalités au sein des organisations et même éventuellement prendre la place de ces programmes pour définir les missions sociales des universités. Pour Benschop, le terme ne provoque pas le bon type de motivation, parce qu'il ne fait pas assez puissamment appel à "notre sens de la justice social» (Benschop 2001: 1166). Pour ces chercheur•euses, entre autres, la préférence institutionnelle pour le terme diversité est le signe d'un manque d'engagement envers le changement, et pourrait même permettre à des organisations telles que les universités de dissimuler l'action des inégalités systémiques. Comme le soutient Himani Bannerji, le langage de la diversité peut avoir une certaine efficacité en tant que «mécanisme de reproduction destiné à gérer une hétérogénéité en réalité conflictuelle » (Bannerji 2000 : 37). La diversité pourrait être interprétée comme l'une des techniques par lesquelles le multiculturalisme de gauche négocie la différence en gérant ses «composants» les plus «encombrants» (Fleras 2011: 1214). La diversité peut ainsi fonctionner comme une stratégie de canalisation.

5 Mon analyse du langage de la diversité doit beaucoup à ces critiques importantes. Elles fournissent non seulement un horizon politique à mon analyse, mais parmi les professionnel•les avec qui je me suis entretenue, beaucoup partageaient également cet horizon : els sont conscient•es (et dans certains cas méfiant•es à l'égard) de l'attrait institutionnel du langage de la diversité. Plutôt que de me focaliser sur la façon dont le langage de la diversité s'implante dans les organisations éducatives, je souhaite examiner comment les langages de la diversité sont mobilisés de plein de manières par différent•es acteurs•rices en leur sein. Mon objectif est de donner un sens à comment celleux qui sont impliqué•es dans le travail de la diversité travaillent (ou dans certains cas, ne travaillent pas) de façon complexe et multiple avec le terme diversité.

\section{La diversité officielle}

6 L'expression diversité officielle ne fait pas seulement référence à l'usage de la diversité par les officiel•les, mais aussi à la façon dont la diversité acquiert le statut de description officielle. Dans cette partie, je m'appuie sur le livre de John Austin, Quand dire, c'est faire ${ }^{5}$ (1975), pour envisager la diversité en tant qu'« acte institutionnel de langage ». Le titre choisi par Austin est évocateur: il suggère non seulement que les mots ont le potentiel d'accomplir des choses, mais aussi qu'il existe des instructions à suivre, et que nous pouvons acquérir le savoir-faire qui nous permettrait d'exercer 
cette potentialité (sous la forme du « comment faire pour »). Austin semble dé-limiter la potentialité des mots en suggérant que certains énoncés accomplissent des choses, et d'autres non. Il fait la distinction entre les énoncés descriptifs ou constatifs, qui rendent compte de quelque chose, et les énoncés performatifs, qui accomplissent quelque chose (même si, comme nous allons le voir, cette distinction ne tient pas). Un énoncé descriptif rend compte de quelque chose, qui peut être vrai ou faux. À l'inverse, un énoncé performatif ne rend pas compte de quelque chose : « il indique que produire l'énonciation est exécuter une action" (Austin 1975: 6) [Austin 1991: 42]. Si les énoncés constatifs peuvent être vrais ou faux, les déclarations performatives sont quant à elles, selon Austin, heureuses ou malheureuses: elles peuvent réussir ou échouer en fonction des circonstances de l'énoncé (par exemple, une excuse serait malheureuse si elle était formulée par quelqu'un•e qui ne serait pas sincère, ou dans le cas où une personne déclarerait $x$ sans avoir l'autorité de faire une telle déclaration).

7 L'analyse d'Austin est restreinte à certains types spécifiques d'actes de langage : ceux qui prennent la forme de la première personne du singulier. Je souhaite étendre les termes de son analyse en prenant en compte ce que j'appelle les «actes institutionnels de langage ». Un acte institutionnel de langage prendrait typiquement la forme de la première personne du pluriel. Quand bien même il pourrait être prononcé par un individu, comme par exemple un•e président•e d'université, cela resterait un acte institutionnel de langage si cette personne parle pour l'institution, ou même en tant que telle ou telle institution. Un discours institutionnel pourrait aussi bien faire des déclarations au sujet d'une institution qu'au nom d'une institution. De tels actes de langage peuvent impliquer de nommer : l'institution est nommée, et en lui donnant un nom, on lui donne également des attributs, des qualités et même un caractère. En formulant des déclarations au sujet de l'institution (en décrivant par exemple l'institution comme ayant certains qualités, telle qu'être diverse), les actes institutionnels de langage peuvent également indiquer la direction d'une action future (en engageant une institution dans un plan d'action). Ils pourraient dire que "l'université regrette», ou simplement que «nous regrettons». Ils pourraient aussi dire que «l'université est diverse » ou juste que «nous sommes divers». Si le premier cas semble indiquer une attitude ou un sentiment, que dit ou fait le second cas?

8 Au premier coup d'œil, il semblerait que l'acte de langage " nous sommes divers " soit un énoncé constatif. Le "rôle ${ }^{6}$ » de la déclaration est de décrire " un état des choses » (Austin 1975 : 1) [Austin 1991 : 37]. Les énoncés de diversité constitueraient ainsi des déclarations vérifiables, dont la valeur réelle pourrait être mesurée. Nous pouvons apprécier - au moins partiellement - l'utilité des données sur l'égalité des chances à partir de la façon dont celles-ci peuvent être employées pour tester la validé de la diversité en tant qu'énoncé constatif. Par exemple, des données sont utilisées pour vérifier si les membres du personnel ou les étudiant•es sont issu•es ou non de milieux variés. Lors d'une discussion au cours d'une rencontre sur l'égalité des chances, nous avons parlé de la possibilité d'utiliser des données pour montrer «à quel point l'université était blanche ». En d'autres mots, nous voulions que les données remettent en cause l'usage de la diversité comme description. Une professionnelle avec qui je me suis entretenue utilisait les données pour révéler que l'université qui l'employait échouait, dans sa "philosophie profonde ", à être une institution dirigée par des personnes issues de la diversité. Cela lui permettait d'exposer clairement que l'université «ne peut en réalité pas se permettre de ne pas endosser un rôle pilote dans 
ces domaines ». En d'autres mots, les données sont utilisées comme une technologie destinée à révéler l'échec de l'université à être à la hauteur de son usage de la diversité comme auto-description: "Ouais, ouais, c'est embarrassant quand ça remonte à la surface ». Les données se transforment en technologie destinée à révéler l'écart entre les descriptions officielles de la diversité et ce qui est mis en place par l'organisation'. Dans [«Commitment as a Non-performative»] le chapitre 4 de On Being Included (Ahmed 2012 : 113-140), j'explore la signification de cet écart entre « dire » et « faire » en ce qu'il nous permet de comprendre le pouvoir institutionnel.

9 Toutefois, l'acte de langage «nous sommes divers » ne prend pas simplement la forme d'un énoncé constatif, notamment parce qu'on ne voit pas clairement ce que décrit le mot diversité. Ce manque de clarté n'est sans doute pas spécifique à la diversité, mais il peut révéler le problème même de la description. Austin montre de quelle manière les énoncés constatifs ne sont pas purs et simples ${ }^{8}$ :

Dès qu'on a saisi que l'objet à étudier, ce n'est pas la phrase mais la production d'une énonciation dans la situation de discours, on ne peut plus guère manquer de remarquer ceci : affirmer, c'est exécuter [perform ${ }^{9}$ ] un acte » (Austin $1975: 139$ ) [Austin $1991: 143^{10}$.

10 Même le fait de déclarer quelque chose peut signifier faire quelque chose. Par exemple, une déclaration peut consister en une affirmation: elle peut affirmer l'existence de ce qui est déclaré. L'effet d'une déclaration va dépendre de la situation dans laquelle elle est faite. Dans des situations différentes, les mêmes mots auront une force différente. En conséquence, il est impossible de faire la différence entre des énoncés au niveau de la phrase. Si quand nous disons, nous faisons (en disant quelque chose ou par le fait de dire quelque chose), alors ce qui est fait par les mots dépend des circonstances dans lesquels ils sont dits. Compte tenu de la préoccupation d'Austin à l'égard des énoncés à la première personne du singulier, les circonstances sont pour lui réduites à ce qu'il appelle la «situation de discours». Dans le cas des actes institutionnels de langage, l'institution n'est pas seulement le sujet et l'objet de la phrase (dans la mesure où l'énonciateur•rice parle pour ou en tant que l'institution tout autant qu'iel parle d'elle), mais constitue également la situation dans laquelle se déroule l'acte de langage. Le recours à un énoncé (ce qui lui permet d'être heureux ou malheureux) dépendrait alors non seulement d'autres acteur•rices (qui peuvent être ou non les destinataires de l'énoncé) mais aussi de la culture institutionnelle, de comment les mots qui sont prononcés circulent ensuite, et de la mesure dans laquelle l'institution est transformée par l'énoncé, si elle l'est.

11 Je suggère ici que les aspects performatifs des énoncés qui semblent en apparence descriptifs s'expliquent mieux si l'on se concentre sur l'institution. Il est possible que ce qui est créé par la description de l'université comme étant diverse, ce soit l'idée même d'une université diverse, qui circule ensuite en tant qu'idée au sein de la communauté décrite. "L'université diverse» devient un objet partagé : si d'autres au sein de l'université répètent la description, alors la répétition de l'énoncé lui donne de la force. Nous pouvons penser le processus en tant que génération d'un public. Michael Warner soutient que l'existence d'un public est caractérisée "par le fait qu'on s'y adresse » (Warner 2002: 67, c'est l'auteur qui souligne). S'adresser à un public, c'est générer un public auquel on peut s'adresser. Warner suggère que la circularité de cette logique est essentielle au phénomène de public. La diversité devient un public par le fait d'avoir été adressée à un public. Le/la président•e d'université qui parle de diversité se retrouve par ricochet à "citer» une convention discursive ${ }^{11}$. La logique 
circulaire de l'adresse est à la base de la diversité en tant que système qui circule. C'est la circulation du mot diversité qui crée l'idée même de "l'institution diverse ». En retour, c'est cette idée qui donne au mot diversité sa circulabilité.

Comme je l'ai suggéré plus tôt, il est important de noter que même si la diversité a gagné en popularité, elle n'est pas pour autant répandue. Le mot diversité semble être partout, mais cette apparence pourrait faire partie de ce qu'il produit. En réalité, le mot diversité est loin d'être partout. Même un rapide coup d'œil aux déclarations de mission type des universités suffit à confirmer ceci : s'il est vrai que certaines universités font explicitement référence à la diversité dans les préambules de leurs déclarations, beaucoup ne le font pas. La diversité apparait généralement sous le libellé spécifique de l'égalité, ou comme un libellé à part entière. [Dans "Equality and Performance Culture ", le chapitre 3 de On Being Included (Ahmed 2012: 83-111)], je propose une réflexion sur le statut des mesures d'égalité comme documents. Ce qui m'intéresse ici, c'est la relation implicite entre la déclaration générale de mission et la déclaration spécifique à propos de l'égalité.

13 Pour citer un exemple, une université a la phrase suivante en ouverture de sa déclaration de mission : «La mission de [xxx] est d'apporter sa contribution à la société à travers la quête de l'éducation, de l'apprentissage et de la recherche au plus haut niveau d'excellence à l'échelle internationale. » La phrase d'ouverture de sa déclaration d'égalité est la suivante: "Dans sa quête d'excellence universitaire, [...] est investie pour l'égalité des chances et engagée dans une approche inclusive et proactive de l'égalité, qui soutient et encourage tous les groupes sous-représentés, promeut une culture inclusive et valorise la diversité. » Ici, la relation entre la mission de l'université et la mission de la diversité est explicite : la diversité devient un moyen de poursuivre sa quête première d'excellence, la diversité devient une technologie conçue pour cet objectif. Ainsi «dans sa » quête d'excellence, l'université valorise la diversité. [Dans le chapitre 3 de On Being Included (Ahmed 2012: 83-111)], je reviens sur la relation entre diversité et excellence. Ce que je veux pointer ici, c'est comment dans le langage officiel, le mot diversité tire sa valeur de ce qui est déjà valorisé. La diversité est incorporée dans la terminologie officielle à partir du moment où elle est en adéquation avec les objectifs de l'organisation. J'ai déjà mentionné comment le fait de mettre le mot diversité en circulation devient un objectif clé du travail de la diversité. Mais la transposition de la diversité sur le plan de la description peut aussi poser des problèmes aux travailleur•euses de la diversité. L'usage de la diversité en tant que description officielle peut être une façon de maintenir les valeurs organisationnelles existantes plutôt que de les transformer.

Lorsque la diversité devient une description routinière, il se peut que ce soit la routine de cette description qui soit reproduite. Il est alors possible que les organisations fassent des déclarations telles que «nous sommes diverses" ou «nous adhérons à la diversité » simplement parce que c'est ce que les organisations disent. Nous pourrions appeler ceci le modèle de la diversité " juste pour la forme ». La diversité devient une convention, ou encore une façon conventionnelle de parler de l'université. La diversité devient un langage ritualisé ou un discours de politesse. Austin soutient que de telles formes de discours «n'ont assurément rien à voir avec les performatifs» (Austin 1975 : 81) [Austin 1991 : 99]. Pour ma part, je suggère que les usages officiels de la diversité peuvent être performatifs dans un sens conventionnel : ils engendrent la convention de 
parler de cette façon. Lorsqu'il devient conventionnel de parler de l'organisation comme étant diverse, alors l'acte de langage entretient une convention.

Lorsque la diversité devient une forme conventionnelle de discours, ce qui est désigné • comme divers $\bullet$ devient moins important•e que le nom de diversité. Au cours d'une rencontre à laquelle j'ai participé en Australie, un•e professionnel•le a parlé de la manière dont la diversité était employée pour qualifier la faune et la flore. La diversité institutionnelle devient de l'éco-diversité. Cette mobilité du mot diversité indique que l'effet de la diversité n'apparait pas clairement, même lorsque celle-ci est entendue comme une convention de langage. Il est possible que la diversité soit plus facilement incorporée dans le discours officiel parce qu'elle peut servir à décrire ou affirmer n'importe quoi. Dans une allocution lors de la conférence annuelle des universités du Royaume-Uni, un président d'université a dit la chose suivante : «Cela m'amène à la question de la diversité. Nos universités apportent une immense contribution au monde. Ces contributions sont le fruit d'une diversité d'institutions. » Ici, la diversité devient une valeur d'un secteur: le secteur est divers parce que ses institutions sont diverses dans le sens où elles diffèrent les unes des autres. En tant que qualité (du secteur, de l'institution, des gens qui composent l'institution, et ainsi de suite), la diversité devient mobile. Il est possible que la mobilité du mot ait quelque chose à voir avec la facilité avec laquelle il peut être incorporé dans le langage officiel.

Quelle que soit la façon dont la diversité est utilisée, elle semble être employée comme un moyen d'accumuler de la valeur, parce qu'elle ajoute de la valeur à quelque chose. Peut-être qu'en ajoutant de la valeur, la diversité crée quelque chose. Un•e responsable du personnel m'a un jour dit: «Et nous avons un corps étudiant très divers, n'est-ce pas? Notre corps étudiant est un corps extrêmement divers. Il vient de tellement de pays différents, entre autres». Cette description nous apprend comment la diversité devient un attribut, ce qui conduit à la création d'un corps singulier (si les étudiant•es viennent de divers pays, alors iels peuvent être appréhendé•es, et donc traité•es, comme un corps divers). En étant attribuée, la diversité crée un certain type de corps. Curieusement, en tant que technique d'attribution, la diversité peut se transformer en contenant : permettant ainsi la présentation du corps comme unifié dans sa diversité.

De cette manière, la diversité fait intervenir l'univers esthétique de l'apparence, ainsi que l'univers moral de la valeur. Elle crée un corps qui peut être vu et valorisé en tant que corps divers. Les descriptions officielles s'intègrent à un ordre moral et esthétique : une apparence de valorisation. Le sens donné à la description officielle réside peut-être dans les officiel•les elleux-mêmes : ce serait alors moins le contenu de l'acte de langage qui donnerait son importance à la diversité que la personne qui parle ou l'autorité au nom de laquelle elle le fait. De nombreux•ses professionnel•les de la diversité ont parlé de la valeur de la diversité lorsqu'elle est évoquée par des officiel•les, qu'iels soit ou non engagé•es pour la diversité : « Les gens savent que si la présidence de l'université ne dit pas les choses appropriées, alors ils n'ont pas à le faire non plus ». La diversité devient alors le fait de "dire les choses appropriées ", de telle manière que le discours officiel produit un pré-requis culturel qui encadre ce qui peut être dit ou non. Quand les officiel•les parlent le langage de la diversité, alors les autres doivent également parler ce langage. Donner de la valeur à la diversité signifierait alors: faire de la diversité une manière appropriée de parler.

18 À partir du moment où la diversité représente la manière appropriée de parler, elle acquiert de la valeur. Un•e professionnel•le remarque: «si la vice-présidence le 
prononce, alors ça cesse d'être un gros mot, non?" Une tâche essentielle des professionnel•les de la diversité consiste à faire en sorte que le mot diversité soit prononcé par les bonnes personnes, pour qu'ensuite le mot puisse ruisseler dans toute l'organisation. Ou comme un•e autre professionnel•le le décrit :

Si iels disent que c'est important, alors les personnes qui suivent dans la chaine et qui sont celles qui font réellement le travail (parce que, soyons honnêtes, ce ne sera jamais la présidence ou la vice-présidence qui travaillera concrètement sur les questions d'égalité, elles ne feront que dire à quelqu'un e d'autre de le faire), les gens en dessous dans la chaine, donc, entendent juste ce message que "oui, c'est important" et que les personnes au-dessus pensent que c'est important. Je pense que c'est mieux si vraiment elles le comprennent aussi, mais pour moi, la chose la plus importante c'est que les gens à la tête disent que c'est important. comprendre que les mots ont une vie institutionnelle. Puisque le travail de la diversité peut impliquer de travailler contre un système institutionnel de défense, il nécessite souvent l'acquisition d'une sensibilité intime aux mots afin de trouver lesquels peuvent " passer au travers ». Les mots se transforment alors en moyens, en outils pour faire quelque chose $\mathrm{e}^{13}$. Travailler avec les mots, c'est travailler à travers leur association : ils peuvent être plus ou moins efficaces selon la façon dont ils sont associés. Le fait de penser en termes d'association nous permet d'interroger les conditions dans lesquelles la diversité est abordée ou le langage de la diversité est employé, en allant plus loin que la notion limitée de situation de communication : lorsque des mots sont à proximité les uns des autres, c'est un signe de leur saturation et donc de leurs associations passées. Ce qui caractérise une histoire affective, c'est qu'elle n'a pas besoin d'être révélée pour qu'elle produise de l'affect. Des proximités existent non seulement entre les mots, mais 
comme je vais le montrer, celles-ci suggèrent également une relation intime entre les mots et les corps.

Presque tou•tes les professionnel•les avec qui j'ai discuté emploient le langage de la diversité. Lors des entretiens, mes questions d'ouverture consistaient simplement à les questionner sur le mot en lui-même : pourquoi est-ce qu'els utilisent ce mot, comment est-ce qu'els l'utilisent, et qu'est-ce qu'il signifie pour elleux? Certain•es professionnel•les ont laissé entendre qu'els employaient le terme diversité parce que c'est le terme en usage. L'attrait pour ce terme pourrait donc indiquer une circularité ou une boucle: nous utilisons le terme de diversité parce que c'est le terme qui est utilisé, et il est utilisé parce que nous l'utilisons. Il est toutefois important de noter que cette circularité n'est pas spécifique à la diversité. Elle caractérise un phénomène de glissement qu'on pourrait simplement qualifier de mode linguistique : en étant utilisés ou non, les mots vont et viennent dans l'usage. Le savoir institutionnel peut ainsi être défini comme une connaissance de la mode: lorsqu'on sait quels mots sont les plus populaires, on sait comment ils peuvent être alignés affectivement les uns avec les autres. Un•e professionnel•le fait l'observation suivante :

Je dirais que diversité est juste utilisé aujourd'hui parce que c'est le terme le plus populaire. Vous savez, on le voit dans la presse, alors pourquoi est-ce qu'on parlerait d'égalité des chances quand on peut juste dire que c'est de la diversité?

C'est à partir du moment où la diversité est «juste utilisée aujourd'hui » qu'on peut « juste dire que c'est de la diversité ». Lorsque les mots sont populaires - évalués selon ce qui se « voit dans la presse »-ils deviennent disponibles et utiles.

L'attrait pour la diversité semble en partie dû à sa nouveauté. Le fait d'utiliser un mot particulièrement récent permet de s'aligner avec le système de valeurs de l'institution, dans la mesure où c'est souvent à cette «nouveauté » qu'est donnée la valeur.

En tant que mot plus récent, diversité fait le buzz : il se pourrait alors que le son qu'on entende, ce soit celui de la façon dont on en a-buse. Potentiellement, donc, le buzz qui entoure la diversité pourrait être une façon de neutraliser d'autres bruits, tels que celui du racisme (pour une analyse du racisme comme bruit, voir Ahmed 2012 : 141-171).

L'histoire de l'arrivée de la diversité est donc également l'histoire d'une récession, de comment d'autres termes ont perdu leur attrait, sont devenus vieux, usés et datés :

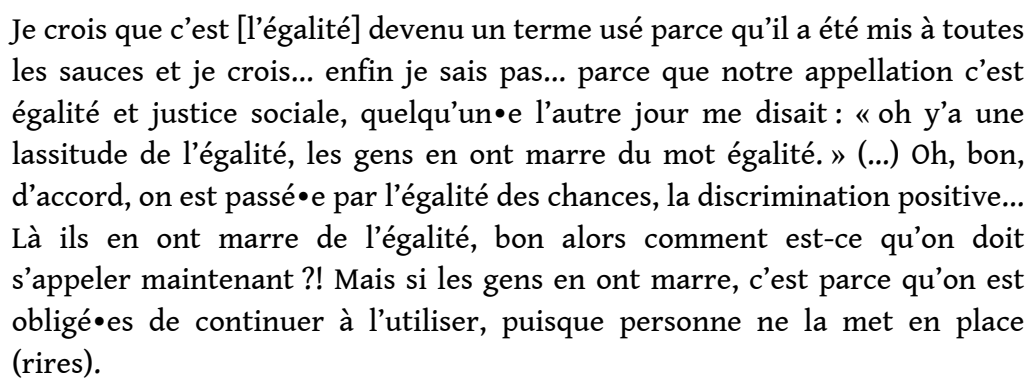

La lassitude, ou même le "ras-le-bol ", vis-à-vis des termes habituels est ici le symptôme d'une certaine réticence institutionnelle: on doit répéter les termes parce qu'ils ne se concrétisent pas, et s'ils ne se concrétisent pas, c'est parce qu'on les répète. Le débat sur la lassitude de l'égalité implique qu'en utilisant des mots moins rebattus, les professionnel•les pourraient être davantage stimulé•es ou être perçu•es comme 
plus dynamiques : « ces termes sont maintenant dépassés et je crois que ça fait un peu l'effet de: "quand y'a un truc qui est dépassé, alors ça donne l'impression que nous on est dépassé•es aussi” ». Si l'on peut devenir dépassé•e par le fait d'être associé•e à des mots dépassés, alors le mot « diversité » offre la possibilité de sembler, ou même d'être, moins dépassé•e. Cette personne continue : « tu te retrouves dans une position où tu te dois de dire ces choses parce que personne d'autre ne les dira. Et les gens ne t'écoutent pas, précisément parce que c'est toi la personne qui les dit.» On continue de les prononcer, parce qu'iels ne les prononcent pas. La fatigue fait partie intégrante de la répétition de cette boucle : c'est parce qu'ils ne produisent pas d'effet qu'on utilise de plus en plus les termes, et s'ils ne produisent pas d'effet, c'est parce qu'on les utilise de plus en plus. Pour ce•tte professionnel•le, le mot diversité offre une possibilité de sortir de cette boucle de répétition. Mais il ne règle pas nécessairement le problème de l'existence même de la boucle. L'implication de cet argument serait alors que certains mots s'alourdissent ou acquièrent un passif à travers leur usage : ils sont lestés par leurs associations. La charge que les mots accumulent semble indiquer que plus ils circulent et moins ils peuvent accomplir de choses. En tant que mot plus léger, diversité peut ainsi accomplir davantage. Bien entendu, il est possible qu'à force de répétition, diversité finisse également par être dépassé. Comme l'a décrit un•e professionnel•le : « Pour l'instant, els n'en sont pas encore lassé•es. J'ai l'impression que c'est un terme qu'iels s'imaginent comme "oh, ouais, diversité!" Iels peuvent s'arranger avec.» L'arrangement avec la diversité pourrait donc être conditionnel: iels n'en sont pas encore lassé •es, pour l'instant ${ }^{14}$.

Les entretiens que j'ai réalisés avec des travailleur•euses de la diversité m'ont permis de comprendre la relation qui existe entre les mots et les corps, la façon avec laquelle certains mots collent à certains corps de telle manière que certains corps peuvent ensuite se retrouver acculés. Comme l'a formulé une professionnelle de la diversité : "Vous savez, dans ce genre de boulot, on se retrouve souvent dans la situation où on commence à dire quelque chose et on voit direct les gens qui se disent : "oh, allez, elle est repartie pour un tour". » Nous avons toutes les deux ri, reconnaissant chacune que l'autre reconnaissait cette scène. Cette scène, tellement familière: je pouvais même voir les yeux se lever au ciel. Cette familiarité provenait en partie de ma propre expérience dans les Women's Studies d'être «la féministe » autour de la table. Mais il y avait quelque chose de plus. Dans The Promise of Happiness (Ahmed : 2010), j'ai écrit au sujet de la figure de la rabat-joie féministe, en pensant à mon expérience de fille féministe assise autour de la table familiale. Autour de cette table, la famille se rassemble. Chacun•e s'installe d'une manière qui semble indiquer qu'iel assure davantage que juste sa place. Quelqu'un•e dit quelque chose qu'on trouve problématique. On répond, calmement, peut-être. On pourrait s'exprimer calmement ou on pourrait aussi bien commencer à s'énerver, reconnaissant par la frustration qu'on est en colère contre quelqu'un•e qui nous met en colère. Quelle que soit sa façon de s'exprimer, celle qui parle en tant que féministe est généralement perçue comme celle qui est à l'origine de la dispute. Encore un dîner de foutu. Dans les institutions, il y a également des tables autour desquelles les corps se rassemblent. La travailleuse de la diversité, un peu comme la rabat-joie féministe, est perçue comme un obstacle à l'espace conversationnel avant même qu'elle ait dit quoi que ce soit. Elle pose problème parce qu'elle persiste à exposer un problème. Encore une réunion de foutue. 
La fatigue qui touche les termes est donc une fatigue institutionnelle : ce qui ralentit les mots, c'est la résistance à leur écoute. Si l'institution a des oreilles, alors elles sont bouchées: les mots ne passent pas au travers. Les moyens développés par les travailleur•euses pour passer au travers deviennent ainsi une stratégie. Une professionnelle suggère que pour des raisons stratégiques, le travail de la diversité devrait être dissocié des formes antérieures de travail pour l'égalité :

Y'a deux ou trois ans, quand j'ai commencé ici, ce bureau était le bureau de l'égalité des sexes. Il se concentrait en priorité sur le personnel, sur les problématiques rencontrées par les femmes dans cet univers professionnel. Le bureau a un peu essayé d'améliorer l'accès des femmes à des domaines d'étude non traditionnels ainsi qu'à des contrats post-doctoraux, mais s'occupait quand même surtout des activités du personnel. Els ont mené certains chantiers vraiment importants, et je ne veux en aucun cas dénigrer mes prédecesseur•euses ni minimiser leur rôle, mais si je veux vraiment être honnête, je crois que leur travail est maintenant un peu dépassé et qu'il a en réalité commencé à être écarté et mis à la marge des préoccupations de l'université.

31 Le «travail de l'égalité » est perçu ici comme dépassé et écarté des préoccupations essentielles de l'université, obsolète et détraqué. Il se pourrait que l'attrait pour la diversité ne soit pas simplement lié au fait qu'il s'agisse d'un mot nouveau, mais également au fait qu'il permette de mettre l'accent sur le nouveau: par exemple, en mettant l'accent sur l'université en tant qu'entreprise au sein de laquelle la nouveauté devient une valeur essentielle ainsi qu'une forme de capital. Le mot diversité s'accorde mieux avec les langages employés pour redéfinir ce que font les universités. Le langage institutionnel peut être interprété comme ayant besoin d'être harmonisé, ou même comme un système d'harmonisation : si le fait d'être incorporé•e dans une institution demande de s'accorder, alors le fait d'être désaccordé•e revient à nuire à l'harmonie de l'institution. Or, ce qui sonne faux sonne trop fort: ça pique les oreilles. La diversité offre ainsi à ses professionnel•les la possibilité de sembler «accordé•es», et donc « en place », par le fait qu'elle ne pique pas les oreilles.

32 Nous pouvons également remarquer la façon dont le «bureau de l'égalité » est évoqué comme un corps physique qui se transforme en corps étranger à l'intérieur même du corps institutionnel. La tâche des professionnel•les de la diversité consiste alors à créer un corps nouveau. Cette travailleuse revendique sa place au sein de l'université en se dissociant du travail déjà réalisé par le bureau au cours de l'histoire. Ce sont des raisons pragmatiques qui sont à l'origine de cette dissociation, bien plus que des convictions idéologiques. Elle décrit la chose suivante: "Nous avions l'impression que les gens étaient juste tellement lassés d'entendre parler de femmes et de harcèlement sexuel.» Pour éviter de lasser les gens, il faut éviter d'utiliser certaines formes de langage. Nous pourrions donc décrire la diversité comme une technique d'évitement: comme une manière d'éviter d'être évité•es. Comme le développe ensuite cette professionnelle :

Il est arrivé qu'on vienne me dire, vous savez, qu'iels pensaient que c'étaient des féminazies, là au bureau de l'égalité, et c'est pourquoi il y avait une grosse résistance, et les gens n'étaient tout simplement pas inclus, et n'étaient pas vus autrement que comme périphériques. La plupart du temps, le bureau n'était pas impliqué dans la communauté universitaire d'une façon très positive. 
Au cours de cet entretien, j'ai été frappée par l'empressement à répéter les stéréotypes associés aux implications réelles de l'action féministe et pour l'égalité (des stéréotypes violents quant à ce travail perçu comme violence) dans le but de créer un nouvel espace pour une forme différente d'action. Cette répétition constitue sans aucun doute un assentiment : un assentiment à l'égard du verdict selon lequel le travail féministe et le travail de l'égalité n'ont pas fonctionné car ils étaient trop extrêmes. Plutôt que de remettre en cause ce verdict, la stratégie consiste donc à produire une image différente. Si l'on pense que ça ressemble à ça, alors il faut modifier la pensée par la création d'une nouvelle image. L'agent•e de la diversité peut "prendre sa place à la table » en ne s'exprimant pas dans un langage problématique, ni dans un langage à problèmes. Modifier l'image du bureau de l'égalité signifie ainsi remettre en cause les méthodes qui ont été auparavant utilisées.

Cette praticienne suggère que la résistance qu'a rencontrée le bureau de l'égalité ne signifiait pas uniquement que l'action pour l'égalité n'était pas incluse dans le travail institutionnel, mais aussi que les autres au sein de la communauté universitaire en général n'étaient pas inclus•es par le bureau de l'égalité. Plutôt que de concevoir ce problème comme un problème institutionnel, il est défini ici comme un problème lié au bureau de l'égalité. Un des objectifs est alors de redéfinir en termes plus positifs la relation entre le travail de la diversité et le travail de l'institution. Comme elle le décrit ensuite :

Si je débarque direct en disant que "je suis là pour transformer vos valeurs", je crains que ça ne soit pas une façon terriblement efficace de développer une relation collaborative de travail.

Le passage du langage de l'égalité à celui de la diversité est alors lié au passage d'un modèle de travail conflictuel à un modèle collaboratif, qui consiste à partager des valeurs plutôt qu'à les faire respecter. En tant que terme, la "diversité » peut ainsi permettre aux professionnel•les de travailler non pas «contre» l'institution, mais «avec» elle. En s'alignant sur l'institution, le travail de la diversité rentre dans les lignes.

36 Il me semble important de signaler ici que l'identification avec l'institution et avec ses valeurs fondamentales peut n'être qu'une impression. Cette praticienne m'a en fait décrit son rôle comme étant celui d'une "travailleuse contre-hégémonique ». Elle redéfinit la relation entre le bureau de l'égalité et l'institution afin de créer l'illusion de travailler avec l'institution, ou d'être sur la même ligne que l'institution, dans l'idée de pouvoir plus efficacement œuvrer contre les normes et les valeurs institutionnelles. En d'autres mots, l'alignement de la diversité sur l'institutionnalité est uniquement maintenu au niveau de l'apparence. Il devient alors possible d'utiliser les termes d'une organisation comme un moyen de se désidentifier de ses normes.

37 Ces désirs de diversité viennent généralement du fait que celle-ci semble désirable : elle s'accorde mieux avec un modèle collaboratif. À partir du moment où le mot diversité est entendu comme étant moins frontal, alors le fait d'employer le langage de la diversité peut être un moyen d'éviter la confrontation. La diversité est plus facilement incorporée par les institutions que d'autres mots, comme égalité, qui semblent en quelque sorte évoquer une politique de critique ou de réclamation vis-à-vis des institutions et de leurs employé•es déjà en poste. La diversité commence alors à être identifiée comme un langage plus inclusif en raison du fait qu'elle n'est pas 
nécessairement liée à un changement des valeurs de l'organisation. En fait, il est même possible que l'inclusivité de la diversité soit justement liée au fait qu'elle ne soit pas associée à l'inclusion des minorités (le langage des «minorités " est plus épineux et associé à un certain type de critique sociale). Je me demande en partie si l'inclusivité apparente de la diversité ne constitue pas autant un désir qu'une description, compte tenu de la facilité avec laquelle dans les discours politiques, comme l'a montré Nirmal Puwar, la diversité tend encore à être associée à celles et ceux qui " ont l'air différent " (2004: 1). Peut-être que la promesse de la diversité peut à la fois être associée à ces corps qui « ont l'air différent » et dissociée de ces mêmes corps en signe d'inclusion (si eux sont inclus par la diversité, alors nous le sommes tou•tes). La promesse de la diversité pourrait donc être décrite comme un problème : le signe de l'inclusion fait disparaitre les signes d'exclusion.

L'attrait que représente le terme de diversité pour certain•es travailleur•euses de la diversité est d'ordre pratique : si le mot est moins menaçant, alors il peut constituer un moyen pour passer au travers des défenses des gens. Comme le décrit un•e professionnel•le :

Je crois que c'est vraiment compliqué : utiliser un terme qui n'est pas acceptable, c'est se retrouver dans l'incapacité de faire quoi que ce soit. D'une certaine façon, si tu veux essayer de travailler avec eux, tu dois utiliser un terme qui ne va pas donner aux gens le sentiment d'être menacés.

La diversité vient remplacer d'autres termes plus « inacceptables » qui peuvent donner aux gens l'impression d'être menacés. [Dans "Speaking about Racism», le dernier chapitre de On Being Included (Ahmed 2012: 141-171)], j'explore comment le fait d'incarner la diversité peut signifier être perçu•e comme une menace, peu importe ce que l'on dit ou fait. Pour l'instant, je dirai simplement que ce mot possède une valeur affective moins négative que d'autres mots, ce qui explique pourquoi il est répété et pourquoi il acquiert une mobilité. Comme l'a décrit un•e autre professionnel•le :

$\mathrm{Tu}$ dois faire en sorte que les choses bougent tout en utilisant la bonne terminologie, histoire que ça rende la tâche un peu plus facile puisque ce n'est pas un mot effrayant.

40 J'y reviendrai en temps voulu, mais le fait que la diversité ne soit pas un mot effrayant fait partie du problème: s'il est détaché de problématiques effrayantes telles que le pouvoir et l'inégalité, alors il devient plus compliqué pour la diversité d'accomplir quoi que ce soit au cours de ses voyages.

41 Que se passe-t-il lorsque les mots que nous utilisons nous permettent d'esquiver les raisons pour lesquelles nous les utilisons? Que se passe-t-il lorsque les mots deviennent confortables ? Comme l'a laissé entendre un•e professionnel•le :

Je vois bien la valeur de la diversité, mais je ne crois pas qu'elle remplace l'égalité des chances. Je crois qu'il s'agit de deux choses différentes qui sont intimement liées, et parfois quand les gens parlent de diversité, ils utilisent un mot derrière lequel il est plus facile de se cacher, plutôt que de réfléchir vraiment sérieusement à comment parvenir réellement à l'égalité des chances.

Le confort de la diversité est ici explicitement défini comme une façon de se cacher, comme un moyen d'éviter d'avoir à réfléchir vraiment sérieusement. Le confort du mot 
diversité peut ainsi permettre aux gens de se sentir plus confortablement installés au sein de l'institution. Nous pouvons ici prendre en considération la critique puissante de Gloria Anzaldúa lorsqu'elle parle de comment la diversité peut être «traitée comme une revêtement superficiel qui ne perturbe en rien aucune zone de confort " (Anzaldúa \& Keating 2009 : 205). Si la diversité constitue une zone de confort, alors elle peut servir de rembourrage pour adoucir les angles de la critique et permettre aux institutions d'être réhabitées comme des espaces plus moelleux.

C'est précisément parce que la diversité, en tant que rembourrage moelleux, est moins négativement connotée qu'elle constitue, au moins pour certain•es professionnel•les, un point de départ, un moyen de passer à travers les défenses des gens. Comme l'a décrit un•e professionnel•le :

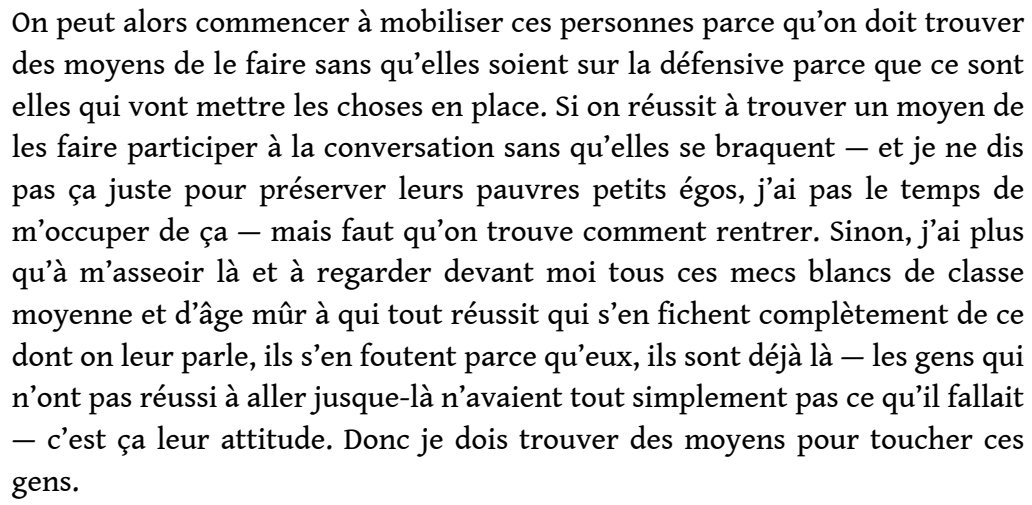

La diversité, c'est "rentrer » et "toucher ", c'est une façon de rentrer en touchant certaines personnes, celles qui autrement ne se sentiraient pas concernées par la diversité en raison de leur propre histoire d'arrivée (une histoire qui peut se transformer en une façon d'occuper l'espace avec le confort de cellui qui est « déjà là »). Il se pourrait que le langage de la diversité soit ce qui permet aux professionnel•les de mettre les gens autour de la table. Une fois qu'ils sont confortablement installés, peutêtre qu'on peut essayer de les déloger en s'occupant de problèmes plus inconfortables.

La circulation de la diversité lui permet certainement d'accumuler de la valeur affective positive. J'ai parlé plus tôt de la différence entre l'acte de langage « nous regrettons » et l'acte de langage "nous sommes divers » qui réside dans le fait que le premier semble indiquer un sentiment ou une attitude. Peut-être que cette différence n'est pas si nette : l'affirmation "nous sommes divers" pourrait également être lue comme une affirmation de sentiment, à travers laquelle le «nous » qui parle, ou dont on parle, est celui qui peut prononcer la promesse affective de la diversité. Nous pouvons interpréter cet acte de langage comme relevant de ce que j'ai appelé un "performatif d'espoir» (voir Ahmed 2010: 200). S'inspirant de la logique promissoire de la psychologie positive (où les sujets se répètent des mots joyeux pour se convaincre qu'ils sont heureux), la diversité en tant qu'acte de langage peut être interprétée comme génératrice de sa propre promesse, comme si l'on pouvait capter la positivité de la diversité en répétant son nom.

La diversité devient un outil positif pour l'action en raison de son statut de terme positif. Nous pouvons ainsi réfléchir à la signification de la positivité en tant que valeur organisationnelle. [Dans le chapitre 3 de On Being Included (Ahmed 2012:83-111)], je pense la diversité en relation avec l'idée de la bonne pratique. Ici, je me concentre sur 
la façon dont les professionnel•les de la diversité envisagent la positivité du terme. Dans certains cas, la positivité de la diversité est associée à son statut de politique d'incitation ou de récompense : la diversité est une sorte de politique du « oui » qui encourage les gens à faire quelque chose, plutôt qu'une politique du "non " qui chercherait à empêcher les gens de faire quelque chose. Comme l'a décrit un•e professionnel•le :

Et c'est un peu comme utiliser la législation comme une banderole pour dire : « vous devez faire comme on vous a dit » et pour jouer un rôle de contrôle. Et vous connaissez le proverbe, on attrape bien plus de choses avec du miel qu'avec du vinaigre. Et je crois que c'est de ça qu'il s'agit. Et celles qui sont de ferventes défenseuses des femmes pourraient me dire, eh bien, tu choisis la facilité. Mais je me vois pas y aller et dire aux gens « vous avez enfreint la loi » et commencer à leur dicter la loi directement sur la table. Ça ne va pas changer leur attitude. On doit faire en sorte que les gens changent leurs façons de penser et d'agir, et en étant inclusives et en essayant de le faire d'une manière acceptable pour les gens, on va pouvoir aller plus loin que si on y allait en disant que "ça ce sont les lois et les règles et si vous ne les respectez pas vous allez vous faire virer ». tant que politique: il ne s'agit pas d'exercer un contrôle ou de «dicter la loi ». La diversité ne menace pas les gens. Pour ce•tte professionnel•le, c'est parce que la diversité n'est pas menaçante qu'elle est plus à même de changer les attitudes. La diversité accumule ainsi de la valeur positive en étant dissociée d'une certaine façon de faire de la politique qui impliquerait de « dicter la loi ».

L'idée selon laquelle la diversité nous permettrait de dépasser la législation a été largement exprimée par les différent•es professionnel•les. La diversité est connotée positivement car elle fournit une motivation à l'action qui ne se fonde pas sur la conformité aux règles. Elle est proactive, plutôt que réactive. Un•e praticien•ne l'exprime ainsi :

Ce que j'en dis moi, de l'égalité, c'est qu'il y a une sorte de cadre légal et je crois qu'on insiste parfois trop là-dessus. Ça crée vraiment une tension parce qu'on doit faire en sorte que les gens soient conscients de la loi, mais on veut aussi aller plus loin que ça, non? On ne veut pas que ce soit juste une question de conformité, alors pour moi, je crois en fait que la diversité est un mot largement plus positif que l'égalité. Pour moi, c'est une célébration. Alors que l'égalité, ça a plus l'air d'être, oh vous savez, presque une question de se conformer aux exigences légales, enfin je sais pas, c'est juste mon point de vue personnel.

La diversité en tant que mode de célébration nous permet donc de dépasser la conformité et d'aller au delà d'une culture de la légalité. Un•e autre professionnel•le décrit les choses ainsi :

Je crois que les gens s'en préoccupent sincèrement parce qu'il y a eu tellement de changements récents et de nouvelles lois, et vous savez, des cas très médiatisés où les choses se sont mal passées. Les gens s'en préoccupent sincèrement, et je crois que ce qu'on essaye de transmettre, c'est que la prise en compte et l'analyse de ces différents paramètres méritent déjà en ellesmêmes de la reconnaissance, c'est une bonne chose à faire en tant que telle. Mais en même temps, on doit reconnaitre que nous avons bien des 
responsabilités légales, que nous devons mettre certaines choses en place, alors c'est en quelque sorte un équilibre, entre la carotte et le bâton presque. Vraiment. Et je crois que parfois, je pourrais dire que certain•es autres collègues sont allé•es trop loin avec le bâton plutôt que d'utiliser la carotte, et que les gens ne réagissent pas toujours bien à ça. le bâton par la carotte, mais que nous devons trouver un équilibre entre les deux. Ce qui est sous-entendu ici, c'est que la diversité, en tant que carotte, pourrait produire une meilleure série de réactions. Là encore, remarquons l'évocation de l'histoire du travail de l'égalité comme ayant été «trop loin avec le bâton ». Est-ce parce qu'elle ne dispose pas de bâton pour "en coller une » si besoin que la diversité «colle » aussi bien? Qu'est-ce qui vient après la conception de la diversité comme miel ou comme carotte ? Ce serait assurément l'association de la diversité au désir : quelque chose que les individus et les institutions pourraient vouloir faire, avoir et être.

Image 1

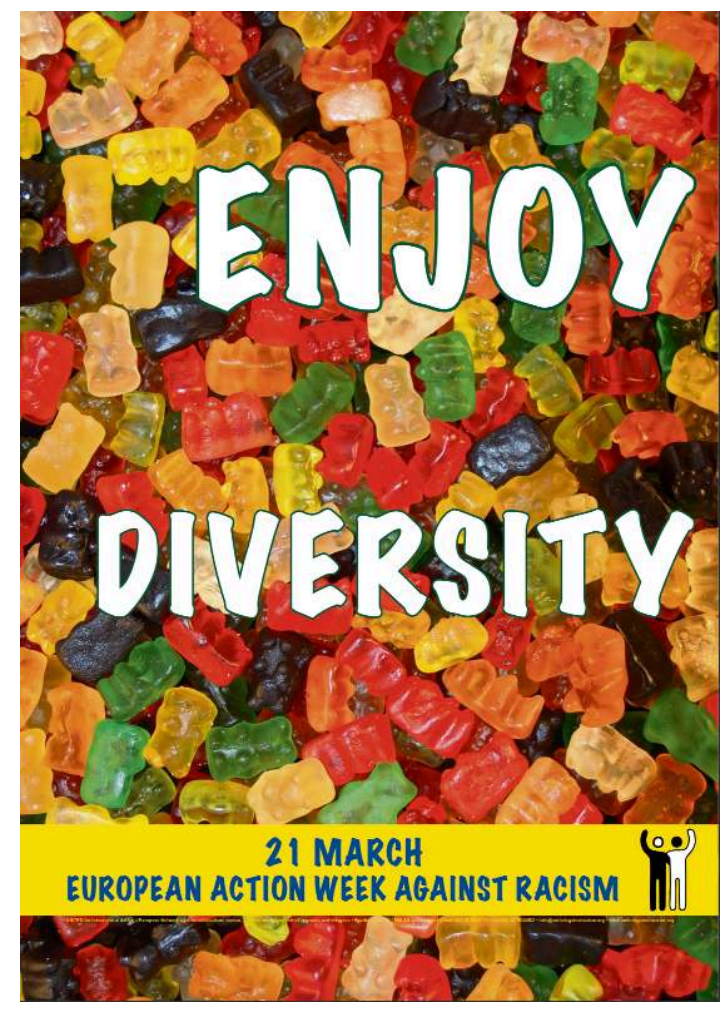

C'est au travers du discours de l'enrichissement culturel - qualifié par un•e enquêté $\bullet$ de modèle de «l'étal du traiteur thaï » - que la diversité apparait clairement comme une politique de la "positive attitude ». La diversité peut être célébrée, consommée et engloutie. Elle peut ainsi être absorbée par le corps de l'université aussi bien que par celui des individus. Pour emprunter l'expression évocatrice de bell hooks (1992), la diversité pourrait en effet même constituer un moyen "d'engloutir l'autre ». La diversité évoque les plaisirs de la consommation. En ajoutant du piquant et de la couleur, les corps des autres "relèvent le plat insipide de la culture blanche dominante " (hooks 1992 : 21). Prenons par exemple l'affiche « Enjoy Diversity » (voir image ci-dessus). La diversité est ici imaginée comme un assortiment de douceurs colorées. La diversité implique-t-elle non seulement l'esthétisation de la couleur mais 
aussi l'exigence que celleux de couleur soient doux•ces? Comme l'a souligné Sirma Bilge, un des slogans utilisés au cours de cette campagne était : « Les racistes n'ont ni courage ni goût $\mathrm{t}^{15}$ ». La diversité est ici narrée comme un plaisir joyeux pouvant nous faire dépasser le racisme, qui pour sa part est réduit à une question de mauvais goût. De leur côté, celleux qui apprécient la diversité ont bon goût. Mais si la diversité constitue une différence digeste, alors les autres formes de différence deviennent indigestes et ne peuvent donc pas être assimilées par le système digestif de l'organisation ${ }^{16}$. L'édulcoration de la diversité (comme sucre, comme miel) pourrait ainsi constituer un moyen d'établir les limites de ce qu'une organisation peut incorporer.

La plupart des professionnel•les avec qui j'ai parlé de manière formelle ou informelle avaient parfaitement conscience de la positivité du terme " diversité » et de ce qui est en jeu dans son attrait institutionnel. Pour certain•es d'entre elleux, c'est sa positivité qui fait de la diversité un outil utile pour que des choses soient mises en place, quelles que soient ces choses. Pour les autres, c'est la positivité du terme qui constitue le problème. Une professionnelle décrit la chose suivante :

\begin{abstract}
Alors maintenant on parle tou•tes de diversité et ça veut dire que tout le monde est différent mais égal, et c'est agréable et tout bisounours, et on peut se sentir bien par rapport à ça et avoir le sentiment d'avoir résolu le problème, alors qu'en réalité on n'est pas du tout proches de le résoudre et on doit, je crois, comprendre que, bon, le concept de diversité s'intègre beaucoup mieux à l'idée que l'université se fait de ce que ça implique d'être la grande bienfaitrice. J'ai invariablement ignoré ce terme, en fait, j'ai même des cartes de visite que j'ai imprimées qui disent "chargée d'égalité » et certaines des commissions l'utilisent et j'ai en quelque sorte juste ignoré le fait qu'elles le font parce que je pense que c'est un concept qui n'est vraiment d'aucun secours. Ce n'est rien d'autre qu'une bonne excuse pour se défiler, ça permet juste aux gens de s'en sortir en pensant que «oh, tout le monde est différent", et de vraiment ignorer les barrières qui sont opprimantes, parce que si on regarde tout le monde comme des individus, alors on peut faire l'impasse sur le fait qu'il existe au sein de l'université du racisme institutionnel, $\mathrm{du}$ sexisme institutionnel, $\mathrm{du}$ validisme institutionnel, etc.
\end{abstract}

53 Pour cette professionnelle, c'est le fait même de parler de diversité qui permet aux individus de se sentir bien, de créer l'impression qu'ils ont « résolu le problème ». La diversité participe ainsi de la création d'une illusion d'égalité, qui cadre avec la mission sociale de l'université : avec l'idée que l'université se fait d'elle-même comme faisant le bien (« la grande bienfaitrice»). La diversité peut ainsi permettre aux organisations de conserver la vision positive qu'elles se font d'elles-mêmes. Elle produit également l'individu en tant qu'objet adéquat: si la diversité correspond à ce que les individus possèdent en tant qu'individus, alors elle donne la permission à celleux qui travaillent dans les institutions de se détourner des réalités en cours de l'inégalité institutionnelle. Cette professionnelle ressent tellement fort la diversité comme " une bonne excuse pour se défiler » qu'elle a ôté le mot diversité de sa carte de visite, alors même qu'elle était embauchée comme responsable de l'égalité et de la diversité. Elle refuse de s'identifier à ce terme et refuse ainsi de renoncer à d'autres termes, comme égalité. D'autres professionnel•les se sont également montré•es sceptiques face à l'attrait de la diversité comme forme de bonheur institutionnel : 
La diversité cache les problèmes. (...) La diversité, c'est comme une grosse pomme rouge et brillante, ok, qui a l'air complètement merveilleuse, mais si on en vient vraiment à découper cette pomme, il y a un trognon pourri à l'intérieur, et là on sait qu'elle est en fait intégralement en train de pourrir et qu'en réalité personne ne s'y intéresse. Tout a l'air merveilleux, mais les inégalités ne sont pas traitées. Pour moi, la diversité, c'est une activité promotionnelle: on peut en tirer quelques bénéfices, mais juste parce que c'est un terme à la mode en ce moment et qu'il peut donc permettre à des gens de commencer à s'impliquer et à chercher à en savoir plus. On se retrouve avec des grosses compagnies comme Ford et toutes ces autres organisations privées qui sont vraiment à fond dans la diversité, vous savez, d'une manière qui permet en elle-même d'influencer d'autres organisations grâce à ce que certaines font. Alors je crois que, bon, j'ai juste la crainte qu'elle n'interpelle pas les réalités des cultures institutionnelles. D'accord, ça pourrait fonctionner pour changer légèrement la culture, mais pas tant que ça.

Par conséquent, si l'on fait la promotion de la diversité, il se pourrait que ce soit parce que cela permette à l'université de faire sa propre promotion, créant ainsi un vernis ou une illusion de bonheur. Nous pourrions simplement appeler ceci le modèle de la « diversité joyeuse », au sein duquel la " parole de la diversité » devient une " parole du bonheur ", comme l'ont décrit Joyce M. Bell et Douglas Hartmann (Bell \& Hartmann $2007^{17}$ ). La diversité fournit une image positive et étincelante de l'organisation qui permet de dissimuler les inégalités, et donc de les reproduire. En écoutant ce•tte professionnel•le, j'ai tout de suite pensé à la critique formulée par Betty Friedan de l'image de la femme au foyer bienheureuse dont le « sourire béat » cache en réalité un malaise (Friedan 1965 : 19-20 ; voir également Ahmed 2010: 50-51). Le sourire de la diversité empêche le «trognon pourri » de faire surface. Ce•tte professionnel•le laisse entendre que la diversité représente un moyen ne pas interpeller les cultures institutionnelles, ou éventuellement de les interpeller seulement « légèrement », ce qui implique que le fait de les interpeller légèrement peut être un moyen de ne pas les interpeller. Nous pouvons revenir à mes commentaires précédents au sujet de la diversité officielle : la diversité est quelque chose dont on parle, créant ainsi l'idée de l'université comme étant diverse. La création d'une idée de l'université comme étant diverse pourrait modifier légèrement l'idée de l'université. En effet, une légère modification de l'idée de l'université pourrait constituer une façon de protéger le cours des choses en dissimulant les choses en cours.

$\mathrm{Si}$, pour certain•es professionnel•les, la positivité du terme diversité le rend utile pour faire asseoir les gens autour de la table, pour d'autres, sa positivité constitue un problème car elle permet de dissimuler les raisons pour lesquelles on pourrait vouloir faire asseoir les gens autour de la table. Nous pourrions décrire cette différence comme relevant de la stratégie. Dans les deux cas, la positivité du mot diversité est un élément crucial dans le choix des mots à utiliser et de la façon de les utiliser.

\section{Les stratégies de la diversité}

Lorsqu'ils deviennent un moyen de parvenir à une fin, les mots sont employés de manière stratégique. Pour certain•es professionnel•les, le mot diversité constitue un moyen pour arriver à une fin: c'est une façon de passer à travers les défenses des 
institutions et des individus. Les choix effectués par les professionnel•les sont bien entendu plus que des choix de mots. Les praticien $\bullet$ nes doivent également plaider des cas : par exemple, pour justifier le besoin d'enclencher des changements de procédure au sein de l'organisation ou dans la façon de mener les commissions, pour obtenir les ressources pour soutenir un projet lié à la diversité (tel que la formation du personnel, par exemple), pour entreprendre des recherches sur l'organisation et son rapport à l'égalité [...]. Plaider un cas, c'est présenter un argument d'une certaine manière, dans un objectif donné.

Lorsqu'els plaident de tels cas, certain•es travailleur•euses de la diversité utilisent le langage généralement employé par l'auditoire auquel els s'adressent. En d'autres mots, els appréhendent leurs choix de langage comme étant déterminés par leurs destinataires. Bien entendu, toute détermination de ce type implique un acte de lecture : les praticien •nes ne se contentent pas d'écouter les mots utilisés par les gens auxquels els parlent, els établissent également leurs propres jugements quant à ce que peuvent entendre différents auditoires. Comme le décrit un•e professionnel•le :

C'est selon ce qui marche. Si une personne peut seulement entendre telle façon de plaider ou tel type de cas, alors on lui parle le langage qu'elle peut entendre. Et pareil pour les autres formes de langage que les autres personnes à qui on parle peuvent entendre. (...) Pour certaines personnes c'est la compassion, parfois c'est l'approche pragmatique, parfois c'est la peur, parfois (...) c'est tout ce qui servira de bon levier pour toucher ce type de personne.

Ici, le travail de la diversité consiste à trouver ce qui fonctionnera pour qui : cela ne nécessite pas seulement de lire le caractère de l'institution [...], mais aussi celui des individus, afin de trouver ce qui sera un "bon levier " pour atteindre différents types de personnes. C'est un processus qui prend du temps et qui nécessite souvent de tester différents mots avant de trouver celui qui sera le bon pour la bonne personne. Comme le décrit ensuite ce•tte même professionnel•le :

On en utilise un, une fois, puis un autre la fois suivante, et encore un autre la fois d'après. On continue de se servir de différents types de discours jusqu'à ce qu'on trouve le bon pour la bonne personne.

On continue d'employer différents discours à différents moments, jusqu'à ce que l'on trouve le bon : et le bon, c'est n'importe quel terme qui fonctionne auprès de l'auditoire avec lequel on travaille.

60 Certain•es professionnel•les parlent du fait de plaider comme d'une forme de traduction: il s'agit de traduire sa plaidoirie en faveur de la diversité en une autre forme de plaidoirie qui aura plus de chance de capter l'attention, comme nous pouvons le remarquer dans l'extrait suivant :

En fonction du public avec lequel je travaille, je place mon discours à différents niveaux. Je veux dire, quand il s'agit d'utiliser tous les moyens possibles pour inscrire le sujet au programme et concrétiser les choses, je fais vraiment la pute. J'en ai rien à faire. Alors si je dois utiliser l'argument de la diversité productive et expliquer qu'on ne peut pas se permettre de perdre des gens de talents à qui ont doit offrir l'opportunité de s'engager avec l'université, ce qui nous permettra de maximiser notre résultat final, si c'est ça que vous voulez, je vais utiliser cet argument, parce que l'effet final sera le même. Si je suis dans une situation où les gens sont plutôt du genre à être 
contre les trucs féministes mais qu'ils sont pour la coopération internationale, je m'en fiche, je vais leur parler des problématiques qui entourent la mondialisation et l'internationalisation, ainsi que de la nécessité d'améliorer notre compréhension des personnes qui nous sont différentes. J'utiliserai ces argumentaires, j'utiliserai ces termes, parce que ce sont les termes qu'ils comprennent. Parce que je sais que si on peut faire en sorte que les gens commencent à mettre en place un programme inclusif, à citer des personnes racisées dans leurs références et à ramener des connaissances d'autres endroits du monde parce qu'ils le font en pensant que ça va améliorer leur réputation internationale et leur servir pour avoir des débouchés pour leurs étudiant•es internationaux•ales, ce qui va se passer c'est qu'ils vont ouvrir leurs espaces à tous les groupes minoritaires. Alors je m'en fiche vraiment. J'utiliserai n'importe quel levier que je jugerai utile pour concrétiser des avancées parce qu'ensuite on peut construire à partir de ça. Si on peut impulser une dynamique, elle s'amplifiera. Mais pour commencer, il s'agit déjà de lancer la dynamique.

61 Cette professionnelle identifie son mandat comme étant destiné «à déclencher un changement culturel » et définit son projet en termes de résultats, d' " effet final » ou de «concrétiser les choses». Elle est prête à utiliser n'importe quel langage qui fonctionnera, afin de présenter ses arguments en des termes qui lanceront «une dynamique ». Cette disposition favorable à utiliser des mots qui fonctionnent laisse apparaitre une sorte d'indifférence (elle s'en fiche, utilise n'importe quel mot). En d'autres mots, aucun mot n'est trop compromettant, du moment qu'il s'agit de mots qui fonctionnent (on peut être une « pute » du langage, dire et faire tout et n'importe quoi). Il me semble important de signaler que cette praticienne est la même que celle qui se décrit comme une travailleuse contre-hégémonique. Lorsqu'une relation au langage est définie en des termes purement instrumentaux, elle peut en fait créer un espace permettant de se désidentifier des mots qui sont employés. On observe un détachement vis-à-vis des mots en eux-mêmes, dans la mesure où ils ne deviennent alors rien de plus qu'un simple moyen de faire des choses. Une question politique qui se pose alors est de savoir jusqu'à quel point nous pouvons nous distancier des mots que nous utilisons. Je considérerais ceci comme une question empirique ouverte, qu'il vaut toujours la peine de se poser à nous-mêmes lorsqu'on travaille tout aussi bien « sur » que "dans " les institutions. Si en disant, nous faisons, alors nos dires peuvent également nous faire quelque chose. Et l'on ne sait pas toujours à l'avance ce que les mots vont faire.

Après cet entretien, j'ai commencé à réfléchir aux stratégies de la diversité en termes de permutation. Les travailleur•euses de la diversité permutent entre différents langages, dans la mesure où différents langages peuvent actionner différentes manettes (une « manette institutionnelle » est ce qui permet d'activer quelque chose). Ce qu'il est intéressant de souligner ici, c'est comment des logiques en apparence contradictoires peuvent être utilisées simultanément: en d'autres mots, le modèle d'entreprise et le modèle militant peuvent être utilisés ensemble, ou alors il est possible de permuter entre les deux en se basant sur un jugement quant à celui qui fonctionne à quel moment et auprès de qui. Cette permutation nécessite de raccorder le mot diversité à d'autres mots, en mobilisant différents types de vocabulaires. Les professionnel•les travaillent avec le terme diversité en le raccordant aux autres mots qui sont déjà valorisés par les différentes entités avec lesquelles els travaillent. 
63 En tant que manettes institutionnelles permettant des permutations, les données constituent également des ressources importantes pour les travailleur•euses de la diversité. Cette même praticienne explique la chose suivante :

Oh, il n'était pas question de dire que nous devrions faire ça parce que ça allait nous apporter un sentiment de chaleur diffus et enveloppant. Il s'agissait plutôt d'aborder ça comme nos indicateurs de performance, d'exposer les raisons de tout ça, de venir avec les épais compte-rendus qui sont exigés, d'expliquer ce qu'on devrait mettre en place et de montrer les données qui viennent étayer tout ça.

64 Les données constituent ainsi une technologie cruciale dans le sens où elles favorisent également la production d'un•e soi compétent•e. Les données sont censées représenter des éléments "solides ", un type de preuve dont la "véracité » est indépendante de toute orientation émotionnelle vis-à-vis du monde (« un sentiment de chaleur diffus et enveloppant »). Évidemment, une telle performance relève de la stratégie : pour être entendu•es, les agent•es de la diversité ne peuvent pas se permettre d'être perçu•es comme étant trop gentil•les, et par conséquent comme présentant une valeur moindre pour l'organisation. L'agent•e de la diversité en vient à incarner la valeur diversité en semblant habiter les valeurs de l'université. En ayant l'air "comme eux", les travailleur•euses de la diversité ne sont plus perçu•es comme des étranger•es mais comme des autochtones, comme des personnes qui sont chez elles dans la culture ambiante de l'université ${ }^{18}$. La professionnalisation du travail de l'égalité et de la diversité nécessite ainsi la production d'un nouveau type de corps associé aux travailleur•euses de la diversité. L'éclat de la diversité serait alors lié non seulement à la promesse de nouveaux mots, mais aussi à celle de nouveaux corps.

Les travailleur•euses de la diversité ont tendance à employer les termes qui sont déjà valorisés par celleux avec qui els travaillent, ainsi que par les institutions au sein desquelles els travaillent. L'alignement avec les institutions n'est pas simplement produit par l'usage du mot diversité, mais également par la juxtaposition de ce mot avec d'autres mots régulièrement employés par ces institutions. La diversité est ainsi rendue plus attrayante en étant associée à l'image idéale que l'université se fait d'elle-même, c'est-à-dire à ce qu'elle imagine déjà comme étant sa mission première ou ses valeurs fondamentales. [...] Cela fait des praticien•nes de la diversité de très bon•nes lecteur•rices de ce que l'on peut appeler le caractère institutionnel.

Dans certains cas, le mot diversité est déjà utilisé dans la façon dont les organisations se vendent et assurent leur promotion. Une professionnelle dit la chose suivante au sujet de son institution: "La diversité fait partie de sa philosophie, elle pourrait même chercher à se vendre de cette façon.» Une autre praticienne décrit son université comme «tirant sa fierté de ses labels d'égalité ». Toutefois, elle indique également que « parfois, ces labels ne sont pas mis en œuvre comme ils devraient l'être ». Ainsi, le fait d'avoir un label d'égalité ou de diversité ne signifie pas nécessairement que quelque chose soit mis en place. $\mathrm{Ou}$, comme un•e autre praticien •ne le formule :

Alors les gens nous voient comme une université égalitaire, mais ça ne veut pas dire qu'on fait réellement quoi que ce soit. En fait, on n'a pas beaucoup de programmes en place ni vraiment de stratégies qui sont mises en œuvre pour recruter des étudiant•es d'origines socio-économiques modestes, comme la plupart des universités doivent le faire, parce que nous, on n'a pas besoin de faire quoi que ce soit. 
Le fait d'être vue comme « étant diverse » peut ainsi être un moyen pour l'organisation de «ne pas créer de diversité », parce qu'il lui suffit de dire qu'elle « l'est » ou qu'elle « le fait » déjà, ce qui signifie qu'elle considère qu'il n'y a rien de plus à faire.

Dans d'autres cas, les professionnel•les de la diversité peuvent chercher à associer le mot diversité aux missions fondamentales de l'université précisément parce que ces missions ne sont pas exprimées en ces termes. Une praticienne indique par exemple qu'il lui est possible d'embrasser les langages positifs de la diversité parce qu'ils cadrent plus facilement avec l'idée de l'université en tant qu'« université internationale ». Elle décrit la chose suivante :

\begin{abstract}
La diversité, c'était quelque chose qu'on avait envie d'adopter. On sentait que la diversité avait une connotation positive. Étant une université internationale, on devait évidemment être de bon•nes représentant•es de la diversité ou sinon autant se tirer une balle dans le pied, d'un point de vue marketing. " Égalité » était aussi un mot qu'on aimait bien. On ne voulait pas de l'égalité des chances, on sentait que cette expression avait fait son temps, et bien qu'il me soit par le passé parfois arrivé d'entendre des collègues défendre ça, on partage le même point de vue quant au fait de ne pas trop s'épuiser à défendre ce genre de truc. Alors on a identifié le mot qu'on considérait comme gagnant, et on est parti•es là dessus. On ne voulait pas de "l'égalité des chances » ou de la "discrimination positive» ou de tous les autres mots qui étaient déjà dépassés, et personnellement, je pensais aussi que..., j'ai fait un peu de RP, et je pensais qu'on s'en sortirait bien mieux avec des nouveaux termes.
\end{abstract}

69 Le fait d'«être internationale" est ainsi associé au fait d'être de bon•nes représentant•es de la diversité. Si l'on «devient de bon•nes représentant•es de la diversité", c'est parce que cela est requis quand on "est une université internationale ». Pour qualifier les langages employés par les organisations lorsque celles-ci se décrivent aux autres dans des termes associés à la valeur, on peut parler de marketing. Comme cette même professionnelle le décrit : «Pour atteindre la réussite économique en tant qu'université internationale, il faut être capable de gérer (à défaut d'un meilleur mot) une variété de personnes, alors si on veut aller à un niveau international, on doit être capable de collaborer avec des citoyen•nes internationaux•ales, et certain •es d'entre elleux sont comme nous, et d'autres pas. » Le terme diversité est en adéquation avec l'idée de l'université comme "étant internationale ». Plus particulièrement, la diversité devient un moyen de s'adresser à certain•es autres, à celleux qui sont «des citoyen•nes internationaux•ales»: si la diversité est une question de variété de personnes, alors cette variété prend certaines formes plutôt que d'autres. Peut-être que celleux qui ne sont pas « comme nous » sont alors celleux qui ne parlent pas le langage de la diversité.

to Le discours de la citoyenneté internationale est effectivement utile : il associe le travail de la diversité à des compétences de traduction entre les cultures. La figure du citoyen ou de la citoyenne international•e pourrait être utilement reliée à celle du voyageur ou de la voyageuse du monde qui, en traversant les frontières nationales et en traduisant à travers les différences, peut se sentir davantage à sa place dans le monde. Norma McCaig, membre fondatrice de Global Nomads International, laisse entendre que : «à une époque où il est devenu impératif d'adopter une vision générale, où les compétences en communication interculturelle, en langues, en médiation, en diplomatie et en management de la diversité sont essentielles, les voyageur•euses du 
monde sont mieux équipé•es 》 (McCaig $\left.1996: 100^{19}\right)$. La diversité peut ainsi devenir une sorte d'équipement, un portefeuille de compétences qui étend la portée générale de sujets individuels.

71 Nous pouvons bien sûr souligner comment la diversité se transforme ici en technologie d'élite, ou en technologie à destination des élites. Homi Bhabha a démontré que les multinationales peuvent affirmer leur «engagement pour la "diversité" » aussi longtemps que la diversité est associée à l'élite. En d'autres mots, aussi longtemps que "la démographie de la diversité est essentiellement composée de migrant•es économiques érudit•es - d'ingénieur•es en informatique, de technicien•nes du domaine médical et d'entrepreneur•euses, plutôt que de réfugié•es, de demandeur•euses d'asile politique ou de pauvres" (Bhabha 2004: xiv). La diversité peut donc devenir un moyen de faire d'avantage, ou de devenir plus avantagé•es, plutôt que de contester les désavantages. Peut-être que si diversité est un terme qui réussit si bien, c'est parce qu'il peut plus facilement être associé à la réussite professionnelle et commerciale. La facilité avec laquelle circule la diversité laisse penser que celle-ci peut accumuler de la valeur commerciale tout autant qu'affective. Toutefois, il ne s'agit pas de dire que le travail de la diversité ne peut fonctionner que de cette manière.

\section{La diversité en conversation}

Dans ce texte, je me suis concentrée jusqu'ici sur la façon dont les professionnel•les de la diversité emploient le langage de la diversité et sur les stratégies et les tactiques qu'els développent pour "passer à travers" les défenses institutionnelles et individuelles. Il est important de noter que les conversations ne sont pas juste formulées en des termes utilitaires, comme un moyen d'accomplir quelque chose, mais qu'elles sont aussi interprétées en tant qu'espace au sein duquel se produit le travail de la diversité. Les professionnel•les de la diversité doivent trouver des façons de participer aux conversations ordinaires au sujet des différents sujets relatifs à l'organisation. Comme l'observe une praticienne :

Il y a des conversations qui ont lieu en ce moment même et qui posent la question de ce que sont nos valeurs, de qui nous sommes en tant qu'université, de si nous mettons effectivement en place toutes les choses que nous disons dans notre déclaration de mission, etc.

73 Elle souligne le fait que c'est au travers de conversations qu'elle a réalisé que même si " la notion d'égalité figure dans la déclaration de mission ", elle n'est pas présente dans « la série de documents qui se sont ensuite enchainés et qui rentraient dans des détails de plus en plus précis et complexes». Si les conversations sont importantes, ce n'est pas simplement parce qu'elles entretiennent la question des missions de l'organisation, mais aussi parce qu'elles révèlent comment ces missions sont ensuite traduites ou non dans les faits. Le fait de parler de diversité dans les conversations en cours " au sujet » des différents "sujets » relatifs à l'organisation devient un élément essentiel pour l'implantation de l'égalité et de la diversité dans ces documents qui se sont enchainés.

Lorsque j'ai commencé cette recherche, je présumais que la vacuité de la diversité était un signe de son manque de valeur et d'utilité politiques. Mais certain•es professionnel•les reliaient justement l'efficacité politique de ce mot à sa vacuité : 
Parce que je crois que la diversité est un mot dont personne ne connait vraiment la signification. L'égalité repose quand même sur l'idée d'être juste, les gens peuvent comprendre ça même s'ils l'interprètent mal, mais si on veut bien commencer à parler de diversité, je me suis rendu compte que les gens réagissent en disant : "bon, qu'est-ce que tu entends par diversité ?" et alors on se retrouve avec des gens qui parlent de valoriser la diversité et de comptabiliser les gens qui ont l'air différent (...), peut-être parce que ce n'est pas encore un concept étriqué. peut être définie de manières relativement différentes. Nous devrions remarquer que certaines de ces définitions semblent plutôt problématiques, bien qu'elles soient répandues («comptabiliser les gens qui ont l'air différent»). Le but du travail de la diversité ne serait ainsi pas de résoudre ce qui est problématique mais de l'inclure dans la conversation. L'expérience de la diversité nous ramènerait donc à la diversité de l'expérience. $\mathrm{Ou}$, comme un•e autre professionnel•le basé•e dans une université australienne l'a décrit :

\begin{abstract}
Je crois qu'avec la diversité, ça évoque juste une variété de tout et de n'importe quoi que l'on fait. On doit l'aborder sous tous les angles. On ne peut pas juste aborder les problèmes à partir d'un environnement anglosaxon de classe moyenne. Ça ne fonctionne juste plus. Ce n'est juste pas ça. Vous savez, ce n'est plus ça le monde réel. Du coup, la diversité c'est vraiment un bon mot pour te faire penser à tous les différents éléments à prendre en compte dans quoi que ce soit que tu pourrais essayer de faire.
\end{abstract}

La diversité peut constituer un tour de passe-passe : elle peut signifier une variété de «tout et de n'importe quoi ». Si la diversité peut faire apparaitre cette variété, alors elle représente un défi aux yeux d'un monde qui rejette la variété, d'un monde qui aborde les problèmes seulement depuis un point de vue particulier. Bien que le fait d'invoquer la variété puisse sembler détacher la diversité de quelque chose en particulier, cette variété constitue un refus de généraliser à partir du particulier (l'environnement anglo-saxon de classe moyenne). C'est alors précisément le manque de référentialité qui devient un point de départ particulier pour une critique de la façon dont on attribue une fonction référentielle à certains points de vue.

Si l'on parle régulièrement de la diversité comme d'un «bon » mot, c'est précisément parce qu'il peut être utilisé de diverses façons, ou même parce qu'il n'a pas de référent :

Nous ne serions bien sur pas opposé•es à changer de nouveau de nom à l'avenir, si nous devions penser à un certain point que cela profiterait à nos actions et à nos objectifs. Mais nous n'avons pas encore trouvé de meilleur mot, et bien que j'aie dit que nous voulions que la notion de justice sociale soit plus fortement présente dans la façon dont nous étions reconnu •es, je n'inscrirais personnellement pas ça dans le titre. En réalité, la diversité s'est avérée être pour nous un très bon mot parce qu'on a pu déverser toutes sortes de choses sous cet intitulé.

Pour certain•es professionnel•les, la diversité est un bon mot parce que différents types d'actions institutionnelles peuvent être incluses sous son nom. Nous pouvons remarquer l'usage du verbe déverser. La diversité est assimilée à un récipient vide. Or, nous pouvons déverser davantage de choses dans un récipient si celui-ci est vide. Si la diversité peut signifier tout ce que l'on veut, alors les professionnel•les peuvent la

GLAD!, 07 | 2019 
définir d'une façon qui leur permet d'effectuer le travail qu'els veulent faire. Bien sûr, il est toujours possible que la diversité soit finalement définie (officiellement, ou part d'autres acteur•rices de l'organisation) de manières qui compromettent ce que les professionnel•les de la diversité veulent en faire. Si les professionnel•les peuvent déverser «toutes sortes de choses sous cet intitulé ", els doivent également collaborer sur ce terme avec d'autres personnes, et s'adapter à l'usage qu'elles en font.

Certain•es professionnel•les laissent toutefois entendre que leur tâche est d'encourager les autres personnes dans l'institution à utiliser la diversité de la bonne façon. L'un•e d'entre elleux dit la chose suivante :

De manière générale, je serais tenté •e de dire que les termes perdent très facilement leur sens. Lorsque leur usage se généralise, ils peuvent se transformer en raccourcis pour certaines questions, si vous voulez. Voilà pourquoi il est important que des termes comme diversité soient placés dans leur bon contexte.

80 Même si les travailleur•euses de la diversité ne peuvent pas déterminer les contextes dans lesquels circule la diversité, els peuvent s'efforcer de lui donner le bon contexte aux moments et aux endroits où elle est effectivement évoquée. Nous pouvons interpréter ce processus comme relevant d'une politique du réattachement: les professionnel•les œuvrent à réattacher à la diversité les significations qu'elle a pu perdre au cours de ses voyages ou à travers ceux-ci. Si le succès de la diversité s'explique en partie par le fait que cette notion a fini par être détachée de l'histoire des luttes contre l'inégalité, alors il se pourrait que le succès du travail de la diversité dépende de sa capacité à réattacher le mot à cette même histoire.

81 À travers ma propre expérience dans la rédaction de règlements en faveur de l'égalité raciale (voir Ahmed 2012 : 83-111), j'ai été frappée par l'importance de la conversation, même lorsque le résultat de la conversation était l'un de ces documents (ce qui semble dans une certaine mesure suggérer que l'intérêt de la conversation résidait ailleurs). Nous parlions entre nous du fait que nous étions un groupe d'employé•es qui étaient tou•tes arrivé•es dans les réunions et dans l'institution de façons très différentes. Nous avions également des relations très différentes aux mots que nous finissions par employer dans les documents que nous rédigions. Dans une certaine mesure, nos conversations affectaient les mots que nous faisions circuler dans les documents : nous incluions le mot diversité, mais nous faisions attention à le mettre à côté d'autres mots, comme égalité, blanchité et racisme. La conversation ne nous a pas amené•es à adopter de définition officielle pour aucun de ces mots. Ce qui importait, c'était la création d'un espace au sein duquel nous pouvions parler des mots eux-mêmes. Les mots sont devenus des lignes de raccordement entre celleux d'entre nous qui s'étaient vu•es attribuer la responsabilité de la traduction.

Je n'ai aucun intérêt à idéaliser de telles conversations. Et pourtant, je pense aujourd'hui qu'elles avaient de l'importance parce qu'elles ont montré que le fait de ne pas décider à l'avance du sens et de la valeur de la diversité pouvait permettre à celle-ci d'être partagée en tant que question. Tout acte de langage est déterminé par les termes ordinaires de son usage. Parler, c'est être impliqué•e dans une communauté de gens qui parlent. C'est suivre des règles ou être influencé•e par les conventions. C'est ce qui permet aux sujets de transmettre des significations, pas simplement à travers ce qui est dit, mais aussi par le déploiement de signaux qui nous permettent de faire les choses ou 
de faire en sorte qu'elles soient faites. Si la diversité est un signal, alors c'est une façon d'orienter ou d'être orienté•e. Il nous faut apprendre ses signes, et apprendre à les suivre. À travers la circulation de la diversité, une communauté peut prendre forme. La diversité ne nous indique pas un référent (un objet partagé qui existerait en dehors du langage), ni même ne crée nécessairement quelque chose qui puisse être partagé. Mais en étant énoncée et répétée dans différents contextes, un monde se dessine autour de la diversité. Parler le langage de la diversité, c'est participer à la création d'un monde.

\section{BIBLIOGRAPHIE}

AHMED, Sara. 2000. Strange Encounters: Embodied Others in Postcoloniality. Londres : Routledge.

AHMED, Sara. 2004. The Cultural Politics of Emotion. Edimbourg : Edinburgh University Press.

AHMED, Sara. 2006. Queer Phenomenology: Orientation, Objects, Others. Durham : Duke University

Press.

AHMED, Sara. 2010. The Promise of Happiness. Durham : Duke University Press.

AHMED, Sara. 2012. On Being Included: Racism and Diversity in Institutional Life. Durham : Duke University Press.

ANZALDÚA, Gloria \& KEATING AnaLouise. 2009. The Gloria Anzaldúa Reader. Durham : Duke University Press.

AUSTIN, John Langshaw. 1970. Philosophical Papers. Oxford : Clarendon Press.

AUSTIN, John Langshaw. 1970. Quand dire, c'est faire. Paris : Seuil. [Traduction de LANE, Gilles]. AUSTIN, John Langshaw. 1975 [1962]. How to Do Things with Words. Oxford : Oxford University Press.

BANNERJI, Himani. 2000. The Dark Side of the Nation: Essays on Multiculturalism, Nationalism and Gender. Toronto : Canadian Scholars Press.

BELL, Joyce M. \& HARTMANN, Douglas. 2007. « Diversity in Everyday Discourse: The Cultural Ambiguities and Consequences of « Happy Talk» » American Sociological Review 72 : 895-914.

BENSCHOP, Yvonne. 2001. « Pride, Prejudice and Performance: Relations between HRM, Diversity and Performance » International Journal of Human Resources Management 12 (7) : 1166-81.

BHABHA, Homi. 2004. The Location of Culture. Londres : Routledge (Classics Edition).

BHATTACHARYYA, Gargi. 1998. «Riding Multiculturalism » in Multicultural States: Rethinking Identity and Difference, BENNETT, David (ed.). Londres : Routledge, 252-66.

BLACKMORE, Jill \& SACHS, Judyth. 2003. « Managing Equity Work in the Performative University » Australian Feminist Studies 18 (41) : 141-62.

BRONSON, Po \& MERRYMAN, Ashley. 2006. « Are Americans Suffering Diversity Fatigue ?». Time, 26 mai 2006. Disponible ici : http://www.time.com/time/nation/article/0,8599,1199702,00.html. 
DAVIS, Angela. 1996. « Gender, Multiculturalism, and Class: Rethinking « Race » Politics » in Mapping Multiculturalism, GORDON, Avery \& NEWFIELD, Christopher (ed.). Minneapolis : University of Minnesota Press, 40-48.

DEEM, Rosemary \& OZGA, Jenny. 1997. « Women Managing for Diversity in a Postmodern World » in Feminist Critical Policy Analysis: A Perspective from Post-Secondary Education, MARSHALL, Catherine (ed.). Londres : Falmer, 25-40.

DERBYSHIRE, John. 2010. « Americans Aren't Racist, They Just Have Diversity Fatigue ». Taki's Magazine. 26 août 2010. Disponible ici : http://takimag.com/article/ americans_arent_racist_they_just_have_diversity_fatigue/.

DERRIDA, Jacques. 1988. Limited Inc. Evanston, IL : Northwestern University Press. [Traduction de WEBER, Samuel].

DERRIDA, Jacques. 1990. Limited Inc. Paris : Galilée. [Traduction de WEBER, Elisabeth].

FLERAS, Augie. 2011. « «Cooling Out Troublesome Constituents» : The Politics of Managing «Isms » in the Antipodes » in Managing Ethnic Diversity, HASMATH, Reza (ed.). Farnham, Surrey : Ashgate, 119-40.

FORTIER, Anne-Marie. 2008. Multicultural Horizons: Diversity and the Limits of the Civil Nation. Londres : Routledge.

FRIEDAN, Betty. 1965. The Feminine Mystique. Harmondsworth : Penguin.

GILLBORN, David. 2008. Racism and Education: Coincidence or Conspiracy?. Londres : Routledge. GUNEW, Sneja. 2004. Haunted Nations: The Colonial Dimensions of Multiculturalism. Londres : Routledge.

HAGE, Ghassan. 2000. White Nation: Fantasies of White Supremacy in a Multicultural Society. Londres : Routledge.

HALL, Stuart. 2000. «Conclusion: The Multicultural Question » in Un/settled Multiculturalisms: Diasporas, Entanglements, Transruptions, HESSE, Barnor (ed.). New York : Zed, 209-41.

HILL, Mike. 2004. After Whiteness: Unmaking an American Majority. New York : New York University Press.

HOOKS, bell. 1992. Black Looks: Race and Representation. Londres : Turnaround.

KANDOLA, Rajvinder \& FULLERTON, Johanna. 1994. Diversity in Action: Managing the Mosaic. Londres : Institute for Personnel and Development.

KIRTON, Gill \& GREENE Anne-Marie. 2000. The Dynamics of Managing Diversity: A Critical Approach. Oxford : Heinemann.

LENTIN, Alana \& TITLEY, Gavan. 2011. The Crises of Multiculture: Racism in a Neo-Liberal Age. Londres : Zed.

LORBIECKI, Anna. 2001. «Changing Views on Diversity Management » Management Studies 32 (3) : $345-61$.

LURY, Celia. 2000. " The United Colours of Diversity » in Global Nature, Global Culture, FRANKLIN, Sarah, LURY, Celia \& STACEY, Jackie (ed.). Londres : Sage, 147-87.

LURY, Celia. 2004. Brands: The Logos of the Global Economy. Londres : Routledge.

MANNUR, Anita. 2010. Culinary Fictions: Food in South Asian Diasporic Culture. Philadelphia : Temple University Press. 
MCCAIG, Norma. 1996. « Understanding Global Nomads » in Strangers at Home: Essays on the Effects of Living Overseas and Coming « Home » to a Strange Land, SMITH, Carolyn D. (ed.). New York : Aletheia Publications, 99-120.

MOOR, Liz. 2007. The Rise of Brands. Oxford : Berg.

PUWAR, Nirmal. 2004. Space Invaders: Race, Gender and Bodies out of Place. Oxford : Berg.

RATTANSI, Ali. 1992. « Changing the Subject ? Racism, Culture and Education » in Race, Culture and Difference, DONALD, James \& RATTANSI, Ali (ed.). Londres : Sage, 11-48.

SARUP, Madan. 1991. Education and the Ideologies of Racism. Stoke-on-Trent : Trentham Books.

STRATTON, Jon \& ANG, Ien. 1998. « Multicultural Imagined Communities: Cultural Difference and National Identity in the USA and Australia » in Multicultural States: Rethinking Difference and Identity, BENNETT, David (ed.). Londres : Routledge, 135-62.

TITLEY, Gavan \& LENTIN, Alana. 2008. « More Benetton than Barricades: The Politics of Diversity in Europe » in The Politics of Diversity in Europe, TITLEY, Gavan \& LENTIN, Alana (ed.). Strasbourg : Conseil de l'Europe, 9-30.

WARNER, Michael. 2002. Publics and Counterpublics. Brooklyn : Zone Books.

WITTGENSTEIN, Ludwig. 2004. Recherches philosophiques. Paris : Gallimard. [Traduction de DASTUR, Françoise, ÉLIE, Maurice, GAUTERO, Jean-Luc, JANICAUD, Dominique, RIGAL, Élisabeth].

WITTGENSTEIN, Ludwig. 2009 [1953]. Philosophical Investigations. Oxford : Blackwell.

\section{NOTES}

1. Dans Ahmed (2012: 114-140), je discute des façons dont nous pouvons interpréter la notion de « volonté institutionnelle».

2. Si l'on considère la mesure dans laquelle la diversité remplace des formules telles que égalité des chances ou justice sociale, on pourrait vouloir questionner les effets de ces remplacements. Le remplacement peut également être interprété comme un moyen d'oublier l'histoire des luttes qui entoure ces termes. Dans le contexte britannique, on peut remarquer qu'une grande partie de la littérature récente relative à la diversité ne fait pas référence aux débats plus anciens sur l'antiracisme et le multiculturalisme dans l'éducation. Pour des exemples de ces travaux antérieurs, voir Sarup (1991) et Rattansi (1992). Pour une discussion plus récente sur le racisme et l'éducation, voir Gillborn (2008).

3. Pour des analyses de la façon dont les styles et les marques émergent sous des formes complexes à la fois sur le plan social et symbolique, voir Lury (2004) et Moor (2007).

4. La lecture critique de la diversité institutionnelle qui est proposée dans ce livre peut être située dans un contexte plus large de littérature sur le multiculturalisme. Stuart Hall soutient que dans son sens substantiel, le multiculturalisme «fait référence aux stratégies et aux règles adoptées pour contrôler ou gérer les problèmes de diversité et de multiplicité qui émergent dans les sociétés multiculturelles » (2000: 209). Sneja Gunew suggère que «le multiculturalisme compose avec le management souvent compromis de la diversité géopolitique contemporaine dans les anciens centres impériaux tout autant que dans leurs anciennes colonies» (2014:15). Pour un autre engagement critique vis-à-vis du multiculturalisme officiel et libéral, basé sur une analyse de documents règlementaires, voir Stratton \& Ang (1998). Cela vaut la peine de signaler que dans de nombreuses parties de l'Europe, le multiculturalisme est maintenant déclaré mort, et même accusé d'être à l'origine du terrorisme et de la désintégration du social, comme cela a été exploré par Lentin \& Titley (2011). Comme je l'ai déjà développé ailleurs, le délaissement du 
multiculturalisme au niveau des règlements officiels au Royaume-Uni a contribué à maintenir en place certaines des idées qui permettaient d'éclairer comment le multiculturalisme était exercé dans les règlements : en particulier, l'usage de la diversité comme agent de liaison, ou la diversité comme moyen d'intégrer ou de créer une culture commune (Ahmed 2004, 2010). L'idée selon laquelle le multiculturalisme insisterait "trop» sur la diversité et conduirait ainsi à la ségrégation est un fantasme rétrospectif destiné à justifier le désengagement politique envers le multiculturalisme, quelle que soit la façon dont celui-ci est entendu.

5. [Titre original : How To Do Things With Words, ndlt.]

6. [Dans le texte original, Austin emploie ici le terme de business, qui a été traduit par rôle dans la version française par Gilles Lane. Bien que la traduction française soit juste dans le contexte du livre d'Austin, on peut toutefois supposer que si Sara Ahmed accentue ici ce terme, c'est également en raison des différents sens et connotations de business en anglais, ndlt.]

7. Il est toutefois important de préciser que les données statistiques ne sont pas toujours utiles. Par exemple, lors d'une conférence sur l'enseignement supérieur à laquelle j'ai participé en 2005, les débats se sont centrés sur les limites des données comme technologie. Comme cela a été signalé par un•e discutant•e : « ce n'est pas en le pesant qu'on engraisse un cochon ». Un•e autre discutant•e a exprimé que "pour pouvoir lire les données, les gens doivent avoir certains moyens et compétences ». Bien que les données puissent être utiles pour saisir les inégalités, elles ne constituent pas une fin en elles-mêmes, car lorsqu'elles sont traitées comme telles, elle peuvent au contraire justement se transformer en obstacle. Si l'on suppose que "la pesée » constitue «l'engraissement", alors le fait de ne pas engraisser peut constituer une façon de peser. Comme d'autres technologies, les données peuvent être utiles dans le cadre du travail de la diversité lorsqu'elles servent à soutenir des arguments, mais pas lorsqu'elles constituent des arguments en elles-mêmes.

8. [Austin $1991: 38-39$, ndlt.]

9. [Les crochets dans la citation sont du traducteur français d'Austin, ndlt.]

10. Austin constate que le problème des déclarations est analogue au problème des énoncés performatifs : «certains problèmes qui se sont récemment posés dans l'étude des déclarations peuvent être interprétés simplement comme des problèmes d'infélicité. » (1970 : 248) L'échec de la distinction entre performatif et constatif contenue dans le travail d'Austin est suivi de l'échec de la distinction entre locutoire, illocutoire et perlocutoire. Ces échecs sont productifs dans le sens où ils démontrent l'impossibilité de localiser le "problème » dans des formes particulières d'acte de langage. Je trouve que certaines des critiques à l'encontre de la distinction faite par Austin entre constatif et performatif ne reconnaissent pas à quel point Austin lui-même admettait l'instabilité de ces distinctions comme source de problèmes et de potentialité.

11. Comme le suggère Derrida, tout acte de langage est une citation : "Un énoncé performatif pourrait-il réussir si sa formulation ne répétait pas un énoncé "codé" ou itérable, (...) si donc (il) n'était pas identifiable en quelque sorte comme "citation" ?» (1988: 18) [1990:45]. Dans Ahmed (2012 : 113-140), je parle de comment Judith Butler développe l'idée de Derrida quant à la relation entre citationnalité et performativité. Ce qui m'intéresse ici, ce n'est pas simplement le fait que l'acte de langage "nous sommes divers" soit une citation, mais aussi la façon dont le succès performatif de l'acte de langage dépend de comment celui-ci est re-cité par les autres au sein de l'institution.

12. Penser les mots comme des chemins nous aide également à comprendre comment ils fonctionnent en tant que systèmes d'orientation : en suivant un tracé, nous sommes dirigé•es dans un certain sens. Dans Queer Phenomenology, j'ai exploré ce que j'ai appelé «le paradoxe de l'empreinte ». J'ai noté la chose suivante : « Un chemin se trace sous l'effet du "foulage" du sol. Nous pouvons interpréter le chemin comme une trace des passages antérieurs. Le chemin est fait d'empreintes - de traces de pas qui "foulent", et qui "en foulant" créent une ligne dans le sol. Lorsque les gens cessent de fouler, il se peut que le chemin disparaisse. Et lorsqu'on voit devant 
nous la ligne d'un chemin, nous avons tendance à la suivre, dans la mesure où un chemin "déblaye" le passage. Ainsi, nous marchons sur le chemin tel qu'il est devant nous, mais le fait qu'il soit devant nous n'est qu'un résultat du fait qu'il ait été foulé » (2006:16). Comme les chemins, les mots sont matériels : une fois qu'ils laissent leur trace, ils déblayent un passage et nous avons tendance à les suivre, et en les suivant, nous sommes dirigé•es dans leur sens.

13. Ce modèle du langage comme outil a été proposé par le philosophe Ludwig Wittgenstein: «Pense aux outils qui se trouvent dans une boîte à outils : marteau, tenailles, scie, tournevis, mètre, pot de colle, colle, pointes et vis. - Les fonctions de ces objets diffèrent tout comme les fonctions des mots» ([1953] 2009: 9) [2004: 32-33]. Wittgenstein souligne qu'il est difficile de distinguer clairement l'effet des mots en eux-mêmes et l'effet recherché par leur utilisation: « leur emploi ne nous apparaît pas si nettement » (10) [33]. Je considère l'utilité des mots en terme d'affect (les associations délicates entre des mots qui « collent » les uns avec les autres).

14. Un phénomène de "lassitude de la diversité » a d'ailleurs été constaté. Ce n'est pas un phénomène particulièrement nouveau, même si aucun•e des professionnel•les avec qui je me suis entretenue n'a utilisé cette expression. La plupart des usages de l'expression « lassitude de la diversité » que j'ai recensés proviennent de sources journalistiques étasuniennes. En 2006 par exemple, Po Bronson et Ashley Merryman ont publié un article dans le Time, intitulé : «Are Americans Suffering Diversity Fatigue?». Iels déclarent : «C'est clair que les gens en ont marre de marcher sur des œufs de peur d'offenser ceux qui ont des croyances, des idées et des modes de vie différents. C'en est devenu épuisant, et ils veulent récupérer et reprendre leur vie. L'idée de la diversité semble avoir usé et épuisé l'accueil qui lui a été fait. C'est maintenant comme un•e invité•e qui se serait attardé•e trop longtemps ». La nature importune de la diversité indique les limites de ce que les gens sont prêts à intégrer, épuisés qu'ils sont par les requêtes de ne pas être racistes qui leur sont adressées. La lassitude de la diversité a également été proposée comme explication alternative à celle du racisme. Par exemple, dans un article intitulé «Americans Aren't Racist, They Just Have Diversity Fatigue » (2010), John Derbyshire soutient la chose suivante: «Se pourrait-il que cette phase de notre histoire arrive à son terme? Dans les débats récents autour de la loi sur l'immigration en Arizona et sur la mosquée de Ground Zero, j'ai l'impression d'avoir détecté un parfum de lassitude de la diversité. Se pourrait-il que l'esprit du député Vaile imprègne encore, en quantité, le public étasunien? Pas un esprit de privilège ou de supériorité raciale, non, et encore moins de "haine", mais de satisfaction à l'égard de ce pays tel qu'il est, avec l'équilibre ethnique qu'il a, et de réticence à consentir à la continuation infinie du changement démographique précipité ?». Lorsqu'en tant qu'explication, la lassitude de la diversité remplace le racisme, elle devient une justification des limites de ce qu'une nation est prête à intégrer. La lassitude de la diversité est décrite ici comme une satisfaction à l'égard d'une situation donnée, où la situation en question devient définissable comme ressemblance. Même si diversité semble être un mot plus léger ou joyeux, guilleret et sémillant, nous pouvons remarquer que cela reste un terme délicat et chargé, qui peut encore être associé à l'arrivée d'autres personnes qui «ne sont pas comme nous", et qui peut ainsi être entendu comme une menace pour la continuation de ce nous. Ce qui semble léger dans un contexte donné peut sembler lourdement chargé de connotations négatives dans un autre. Pour une plus ample discussion sur la légèreté et de la lourdeur des mots, voir également Ahmed 2012 (141-171).

15. Communication personnelle avec l'autrice (via Facebook). Je souhaite exprimer ma reconnaissance envers Sirma Bilge, qui a attiré mon attention sur l'affiche Enjoy Diversity et qui a formulé des remarques avisées au sujet de la diversité comestible. Le slogan mentionné peut être retrouvé en ligne sur le site web de United for Intercultural Action: http:// www.unitedagainstracism.org/publications/diversity/.

16. Pour une analyse plus détaillée de la distinction entre différences digestes et indigestes, ou assimilables et inassimilables, basée sur le travail de bell hooks, voir le chapitre 4 de Ahmed (2000). Je traite également de comment le multiculturalisme, en tant que corps national, 
«assimile certain•es autres » en définissant les limites de ce qui peut être digéré. Il y a une longue histoire derrière ce que l'on pourrait appeler le «multiculturalisme de consommation ", au cours de laquelle l'analogie principale se fait entre multiculturalisme et nourriture. Le plat national est conçu comme une façon de mélanger des ingrédients, et les corps des autresracisé•es deviennent les ingrédients à mélanger. Angela Davis décrit la chose suivante : «Le melting pot a été remplacé par la métaphore de la salade. Une salade se compose de nombreux ingrédients et constitue un joli plat coloré destiné à être consommé par quelqu'un•e. On commence donc maintenant à se poser la question de qui consomme le multiculturalisme » (1996: 45). Voir également l'analyse formulée par Ghassan Hage de la «célébration de la diversité » par la métaphore du ragoût dans le contexte australien (2000:119-123), ainsi que celle d'Anita Mannur sur comment le multiculturalisme «accorde un statut particulier» à la nourriture (2010: 224).

17. En tant que discours managérial, nous pouvons relier la "diversité joyeuse » au plus large discours social du multiculturalisme comme "positive attitude». Voir à ce sujet l'analyse convaincante que fait Gargi Bhattacharyya de ce discours, dans laquelle elle montre comment l'obligation de ressentir des sentiments positifs à l'égard de la ville multiculturelle nécessite que le racisme ne rentre pas dans l'équation. Elle note la chose suivante: «Dans l'Angleterre des années 1990, une version de la traduction du multiculturalisme est l'affirmation selon laquelle le fait d'avoir des sentiments positifs à l'égard de sa ville signifie se sentir à l'aise avec des personnes différentes. Ici, contrairement à certains appels locaux à la mobilisation, le racisme ne peut pas être intégré au tableau» (1998: 258). Pour une critique plus récente du multiculturalisme comme " politique de la positive attitude », voir Fortier (2008).

18. Cependant, la décision stratégique de "devenir autochtone" n'est pas sans risques ni limitations. Par exemple, une membre d'une commission pour la diversité m'a parlé de comment elle n'arrivait jamais à joindre la personne responsable de la diversité car celle-ci était en permanence en conversation avec les dirigeant•es et les gérant•es. La réorientation des travailleur•euses de la diversité comme ami•es de l'institution peut ainsi impliquer qu'els deviennent moins attentif•ves aux membres de l'institution qui en sont les plus critiques.

19. Global Nomads International a un «village virtuel » sur : http://www.gnvv.org. L'expression «voyageur•euse du monde » [global nomad, ndlt] est principalement utilisée en référence aux enfants «qui travaillent, ou ont travaillé, dans le corps diplomatique, l'armée, le commerce international, les organismes missionnaires et les agences de service social intergouvernemental ou volontaire, telles que l'ONU ou le Corps de la Paix, ou diverses autres organisations ». Cette description provient de http://www.globalnomads-dc.org. Pour une analyse plus approfondie des voyageur•euses du monde, voir Ahmed (2000 : 84-86).

\section{RÉSUMÉS}

Basé sur des entretiens effectués avec des travailleur•euses de la diversité au sein de l'enseignement supérieur au Royaume-Uni et en Australie, ce texte pose la question des effets de la diversité. Les chercheur•euses féministes et décoloniaux•ales ont offert de puissantes critiques du langage de la diversité. Cet essai souhaite contribuer au débat en examinant comment les travailleur •euses de la diversité travaillent avec le terme diversité au sein du monde éducatif. Ce texte montre qu'en tant que terme, la diversité est utilisée de manière stratégique par ses praticien•nes en réponse à ce qui a été qualifié de « lassitude de l'égalité » : c'est un mot 
plus facilement compatible avec les principes actuels des organisations, voire même avec leur fierté. Ce qui fait l'utilité de la diversité fait aussi ses limites : elle peut se retrouver détachée de l'histoire des luttes pour l'égalité. Ce texte explore la façon dont les praticien•nes doivent réattacher le terme diversité à d'autres mots (tels que égalité et justice) qui évoquent pour leur part cette histoire. L'objectif des travailleur•euses de la diversité est que les institutions s'engagent en faveur de la diversité. Cependant, la signification de cet engagement dépend encore de la façon dont la diversité circule en tant que terme au sein des organisations.

This article asks the question, "what does diversity do?" by drawing on interviews with diversity practitioners based in higher education in the United Kingdom and Australia. Feminist and postcolonial scholars have offered powerful critiques of the language of diversity. This essay aims to contribute to the debate by examining how diversity workers work with the term diversity within the context of education. It shows that diversity as a term is used strategically by practitioners as a solution to what has been called "equity fatigue"; it is a term that more easily supports existing organizational ideals or even organizational pride. What makes diversity useful also makes it limited: it can become detached from histories of struggle for equality. The article explores how practitioners have to re-attach the word diversity to other words (such as equality and justice), which evoke such histories. Diversity workers aim to get organizations to commit to diversity. However, what that commitment means still depends on how diversity circulates as a term within organizations.

\section{INDEX}

Thèmes : Recherches

Keywords : diversity, language, inclusion, racism, equality, institutions

Mots-clés : diversité, langage, inclusion, racisme, égalité, institutions

\section{AUTEURS}

\section{SARA AHMED}

Sara Ahmed est une écrivaine et chercheuse féministe indépendante. Elle a enseigné les Women's Studies à l'Université de Lancaster, et les Race and Cultural Studies à Goldsmiths, Université de Londres. Ses travaux s'intéressent à la façon dont le pouvoir est vécu et contesté dans la vie quotidienne et au sein des cultures institutionnelles. Elle travaille actuellement à l'écriture d'un livre sur la notion de réclamation. Ses précédentes publications incluent notamment: What's the Use? On the Uses of Use (2019), Living a Feminist Life (2017), Willful Subjects (2014), On Being Included (2012), The Promise of Happiness (2010), Queer Phenomenology (2006), The Cultural Politics of Emotion (2014, 2004), Strange Encounters (2000) et Differences that Matter (1998). 